Clean Manuscript for Applied Energy (APEN-D-15-05239)

This paper was presented at the $7^{\text {th }}$ International Conference on Applied Energy (ICAE2015), March 28-31, 2015, Abu Dhabi, UAE (Original paper title: "Study on thermoelectric-hydraulic performance of longitudinal vortex generators in a large-scale thermoelectric power generator" and Paper No. 157).

\title{
Numerical study on thermoelectric-hydraulic performance of a thermoelectric power generator with a plate-fin heat exchanger with longitudinal vortex generators
}

\author{
Ting Ma ${ }^{a, b}$, Xing Lu ${ }^{a}$, Jaideep Pandit ${ }^{b}$, Srinath V. Ekkad ${ }^{b}$, Scott T. Huxtable ${ }^{b}$, \\ Samruddhi Deshpande ${ }^{\mathrm{b}}$, Qiu-wang Wang ${ }^{\mathrm{a}, *}$
}

${ }^{a}$ Key Laboratory of Thermo-Fluid Science and Engineering, MOE, Xi'an Jiaotong University, Xi'an, Shaanxi 710049, China

${ }^{\mathrm{b}}$ Department of Mechanical Engineering, Virginia Tech, Blacksburg, VA 24061, USA *Corresponding author. Tel.: +86(29)82665539; Fax: +86(29)82663502; E-mail address: wangqw@mail.xjtu.edu.cn (Q.W. Wang).

\begin{abstract}
In this paper, the effect of longitudinal vortex generators (LVGs) on the performance of a thermoelectric power generator (TEG) with a plate-fin heat exchanger is investigated. A fluid-thermal-electric multi-physics coupled model for the TEG is established on the $\mathrm{COMSOL}^{\circledR}$ platform, in which the Seebeck, Peltier, Thomson, and Joule heating effects are taken into account. The equivalent thermal-electrical properties of the thermoelectric (TE) module are used in the numerical simulation. The results indicate that the LVGs produce complex three-dimensional vortices in the cross section downstream from the LVGs, thus enhancing the heat transfer and electric performance compared to a TEG without LVGs. Under baseline operating conditions, the heat input and open circuit voltage of the TEG with LVGs are increased by $41 \%-75 \%$ compared to a TEG with smooth channel. The simulations also show that the Reynolds number and hot-side inlet temperature have significant
\end{abstract}


effect on the net power and thermal efficiency of the TEG, but the cold-side temperature has a smaller effect. Additionally, the performance of the TEG under a constant heat transfer coefficient boundary condition is almost the same as the performance under a constant temperature boundary condition. Overall, this work demonstrates that LVGs have great potential to enhance the performance of TEGs for waste heat recovery from vehicle exhaust.

Keywords: thermoelectric power generator; longitudinal vortex generator; equivalent thermal-electrical properties; heat transfer enhancement; plate-fin heat exchanger

\section{Nomenclature}

$$
\begin{aligned}
A & =\text { Cross sectional area of TE leg or TE module, } \mathrm{m}^{2} \\
c_{\mathrm{p}} & =\text { Specific heat, } \mathrm{J} / \mathrm{kg} \cdot \mathrm{K}) \\
D_{\mathrm{h}} & =\text { Hydraulic diameter, } \mathrm{mm} \\
\mathbf{E} & =\text { Electric field intensity vector, } \mathrm{V} / \mathrm{m} \\
I & =\text { Electric current, } \mathrm{A} \\
\mathbf{J} & =\text { Electric current density vector, } \mathrm{A} / \mathrm{m}^{2} \\
k & =\text { Thermal conductivity, } \mathrm{W} /(\mathrm{m} \cdot \mathrm{K}) \\
m & =\text { mass flow rate, } \mathrm{kg} / \mathrm{s} \\
L & =\text { Length, mm } \\
N & =\text { Number of TE legs } \\
p & =\text { Static pressure, } \text { Pa } \\
P_{\mathrm{o}} & =\text { Total power output, } \mathrm{W} \\
Q_{\mathrm{h}} & =\text { Hot-side heat transfer rate, } \mathrm{W} \\
R & =\text { Electric resistance, } \Omega \\
R e & =\text { Reynolds number } \\
T & =\text { Temperature, } \mathrm{K} \\
U & =\text { Area-averaged velocity, } \mathrm{m} / \mathrm{s} \\
\mathbf{u} & =\text { Velocity vector, } \mathrm{m} / \mathrm{s} \\
V & =\text { Voltage, } \mathrm{V} \\
V_{\mathrm{oc}} & =\text { Open circuit voltage, } \mathrm{V} \\
\dot{V} & =\text { Volumetric flow rate, } \mathrm{m} / \mathrm{s} \\
W_{\Delta p} & =\text { Net power, } \mathrm{W} \\
& =\text { Pumping power, } \mathrm{W} \\
&
\end{aligned}
$$

Greek

$$
\alpha=\text { Seebeck coefficient, } \mathrm{V} / \mathrm{K}
$$


$\rho=$ Density, $\mathrm{kg} / \mathrm{m}^{3} ;$ Electrical resistivity, $\Omega \cdot \mathrm{m}$

$k=$ Thermal conductivity, $\mathrm{W} /(\mathrm{m} \cdot \mathrm{K})$

$\sigma=$ Electrical conductivity, $\mathrm{S} / \mathrm{m}$

$\mu=$ Dynamic viscosity, $\mathrm{Pa} \cdot \mathrm{s}$

$\eta=$ Thermal conversion efficiency

Subscripts

$$
\begin{aligned}
\mathrm{c} & =\text { Cold side } \\
\mathrm{E} & =\text { Equivalent thermal-electrical properties for a TE module } \\
\mathrm{f} & =\text { Fluid } \\
\mathrm{h} & =\text { Hot side } \\
\mathrm{i} & =\text { The } i^{\text {th }} \mathrm{TE} \text { leg } \\
\text { in } & =\text { Inlet } \\
\text { leg } & =\text { a single TE leg } \\
\mathrm{o} & =\text { Outlet } \\
\mathrm{s} & =\text { Solid }
\end{aligned}
$$

\section{Introduction}

As a solid-state energy converter, a thermoelectric (TE) material can directly convert thermal energy into electrical energy without additional power generation devices. Poor efficiency for TE devices has limited their competitiveness with vapor compression systems, such as in air-conditioning or heat pump applications. However, the TE devices have been used in smaller-scale applications such as in automobile seats, night-vision imaging systems, and electrical-enclosure cooling [1]. Additionally, many recent advances in materials research have given promise for improved TE devices in the near future. For example, the dimensionless figure of merit in bismuth antimony telluride (BiSbTe) bulk alloys has been increased from 1.0 to 1.4 at $100^{\circ} \mathrm{C}$ through the use of nanostructuring [2]. Using similar nanostructured TE materials, the efficiency of solar thermoelectric generators has shown to be 7-8 times higher than the traditional flat-panel solar thermoelectric generators (TEGs) [3]. Recently, TE devices have attracted considerable attention in the automobile industry since $70 \%$ of the energy released from the fuel is lost in the form of waste heat through the exhaust 
gases or cooling system [4]. Many automotive manufacturers and researchers are exploring the use of TEGs to convert some of the waste heat from the exhaust gas into useful electric power. Hsiao et al. [4] investigated the feasibility of applying TE modules to the exhaust pipe and radiator in an automobile, and showed that a maximum power density of about $50 \mathrm{~mW} / \mathrm{cm}^{2}$ was possible. In et al. [5] examined the performance of a TEG heated by the exhaust gas from an actual engine under various thermal conditions. Their work indicated that the temperature difference between the hot and cold ends of TE module and the differential pressure of the exhaust gas had significant effects on the power generation performance of system. Liu et al. [6] proposed a "four-TEGs" system for the automobile, and a maximum power of $944 \mathrm{~W}$ was obtained in the revolving drum test for a temperature difference of $240^{\circ} \mathrm{C}$. Favarel et al. [7] optimized the thermoelectric systems from the view of examining the position of the TE couples or occupancy rate along the system. The result showed that the maximum output electrical power was obtained for a configuration where TE modules did not completely cover the hot heat exchanger. Liang et al. [8] conducted a numerical simulation to study the performance of a two-stage TEG using the exhaust gas of an internal combustion engine as the heat source, and showed that the peak output power and conversion efficiency had a strong dependence on the thermocouple ratio. Yu et al. [9] analyzed the transient behavior of a TEG capturing heat from the vehicle exhaust under different start-up modes. Their results indicated that a higher vehicle speed could accelerate the transient response. Chen et al. [10] optimized the TEG performance using the Taguchi method, and showed that the output power of the TEG system could be further enhanced by around $6 \%$ by optimizing a second-stage TEG.

In addition to the performance of the TE material, the TE module and system, the thermo-hydraulic performance of the heat exchangers also plays an important role on the overall efficiency of TEGs. Crane and Jackson [11] conducted a numerical simulation to optimize the thermoelectric waste heat recovery system with air cooling in a cross flow heat exchanger. They found that the optimal configuration was obtained at intermediate cooling air and hot fluid flow rates due to increasing power 
losses from the air fan and fluid pump at high flow rates. Lu et al. [12] performed an experiment to test the thermal uniformity and pressure drop of the muffler-like exhaust heat exchangers with different structures, and quantified the interdependence of the flow rate, pressure drop, and temperature distribution. Zhou et al. [13] examined the effect of flow types, hot stream inlet temperatures, pressure drops, cross sectional area, channel length and number of channels on the thermoelectric-hydraulic performance of TEG. Their work demonstrated the complicated nature of system optimization, and showed the importance of having a well-mixed exhaust gas stream in order to promote high heat transfer.

Recently, many groups have examined a variety of methods for enhancing the heat transfer to and from the TE devices in order to improve the overall efficiency of the TEG systems. Lesage et al. [14] and Amaral et al. [15] applied turbulating inserts into the fluid channel in a liquid-to-liquid TEG. These studies showed that panel inserts could enhance the power up to $110 \%$ [14]. However, there was an upper flow rate threshold beyond which the pressure drop caused by the flow impeding inserts might offset its power enhancement [15]. Reddy et al. [16] conducted a numerical simulation to compare the thermoelectric performance of an integrated TE device with rectangular, round end slots, and circular flow channel designs. Their results showed that the integrated TE device with a circular flow channel had better performance than those with round end slots and rectangular flow channels under fixed operating conditions. Metal foams have also been used to enhance heat transfer in a TEG with a plate heat exchanger [17]. This work showed that metal foams with large porosity can effectively increase heat transfer without a significant pressure drop penalty. Pandit et al. [18] examined three-dimensional partial pin fin arrays on the hot-side walls in a gas-to-liquid TEG. The results indicated that the diamond pin fins had the best heat transfer performance, and lower pin-fin channel heights with $50 \%$ clearance provided significantly higher heat transfer coefficients.

Longitudinal vortex generators (LVGs) have also attracted much attention for heat transfer enhancement in both plate heat exchangers and tube-and-fin heat exchangers. Liu et al. [19] found that the LVGs could improve the heat transfer 
performance of a rectangular microchannel by $9-21 \%$ in laminar flow, and $39-90 \%$ in turbulent flow compared to flow in a smooth microchannel. In a rectangular mini-channel, LVGs have also been shown to enhance the heat transfer rate by $10-45 \%$ when the flow velocity ranged from $0.5 \mathrm{~m} / \mathrm{s}$ to $3.4 \mathrm{~m} / \mathrm{s}$ [20]. He et al. [21] proposed a "V" type LVG for a plain-fin round-tube heat exchanger. The results showed that the air-side heat transfer coefficients were increased by $25-55 \%$ for the $30^{\circ}$ and large pair LVGs. In our previous work, the feasibility of using LVGs to improve heat transfer in a small-scale integrated thermoelectric device (ITE) was studied [22]. That work demonstrated that that the heat input, net power and thermal conversion efficiency performance of the TEGs with LVGs could be enhanced by $29 \%-38 \%, 90 \%-104 \%$ and $31 \%-36 \%$, respectively, compared to the ITE with a smooth flow channel. However, the maximum net power of this small-scale ITE was only $0.134 \mathrm{~W}$. In large-scale TEGs, plate-fin heat exchangers are widely used. To the best of our knowledge, LVGs have not been used to enhance the heat transfer performance of plate-fin heat exchangers in the open literature. Most researchers use plate-fin heat exchangers without LVGs in traditional TEGs. In this paper, we propose to use the LVGs to enhance the heat transfer performance of plate-fin heat exchanger and to further improve the thermal-electrical conversion performance of a TEG system. Thus, in this study, the effects of LVGs on the thermoelectric-hydraulic performance of large-scale TEGs with plate-fin heat exchangers are examined.

\section{Physical model and boundary description}

Figure 1 shows the baseline TEG model with a smooth channel for the hot exhaust gas. The hot-side flow channel is formed by a traditional plate fin channel that does not contain any ribs. The length of the plate fin channel is $138.56 \mathrm{~mm}$, the height is $10 \mathrm{~mm}$, the fin pitch is $21.5 \mathrm{~mm}$, and the fin thickness is $1.5 \mathrm{~mm}$. In the model, the inlet and outlet of the hot-side channel are extended to be 5 times and 15 times, respectively, the height of the hot-side channel to support uniform flow for the inlet and to suppress backflow at the outlet. Cold water flows in the cold-side flow channel on the opposite side of the TEG. The wall temperature of the cold-side flow channel is 
regarded as a constant temperature since the liquid has a much larger heat transfer coefficient than the air on the hot-side flow channel. All of the plates as well as fins in the hot-side and cold-side flow channels are made of stainless steel. The thickness of hot-side and cold-side plates is $1 \mathrm{~mm}$.

The TE module is composed of a ceramic substrate, conductive copper, couples of p-type and n-type legs, conductive copper and another ceramic substrate from top to bottom as shown in Fig. 1. The bottom and top surfaces of the TE modules are attached to the cold-side and hot-side plates, respectively. The assembled TE module has a square cross section of $40 \mathrm{~mm} \times 20 \mathrm{~mm}$ with a height of $4.2 \mathrm{~mm}$. There are three TE modules laid between the hot-side and cold-side plates. Each TE module consists of 63 couples of p-type and n-type TE legs, which are made of $\mathrm{Bi}_{2} \mathrm{Te}_{3}$. Each p-type or n-type TE leg has a square cross section of $1.4 \mathrm{~mm} \times 1.4 \mathrm{~mm}$ with a height of $1.6 \mathrm{~mm}$. The thickness of the ceramic and copper layers is $0.7 \mathrm{~mm}$ and $0.6 \mathrm{~mm}$, respectively. Since the main objective of this work is to investigate the effect of LVGs on the performance of the TEG compared to the traditional plain plate-fin heat exchanger, the physical properties of the TE materials are assumed to be temperature-independent, as shown in Table 1. This assumption simplifies the calculations and reduces the computational time.

Because the major thermal resistance is produced at the hot-side channel, the LVGs are proposed to be mounted in the plate fin channel in order to further improve the heat transfer performance, and thus increasing the power output, as shown in Fig. 2. The size of each LVG is $5 \mathrm{~mm}$ (length) $\times 1 \mathrm{~mm}$ (width) $\times 20 \mathrm{~mm}$ (height) with an inclined angle of $45^{\circ}$. The longitudinal pitch and transverse pitch of the LVGs are $34.64 \mathrm{~mm}$ and $4.22 \mathrm{~mm}$, respectively. There are four rows of LVGs mounted along the streamwise direction of the hot-side channel.

All the physical properties for both the fluid and solid materials are assumed to be temperature-independent. For the hot-side flow channel boundary, the mass flow rate and uniform temperature are specified at the inlet, while a pressure outlet boundary at $0 \mathrm{~Pa}$ is used at the outlet. In the baseline operating condition, the inlet velocity of the hot-side exhaust gas in the plate fin channel is $2 \mathrm{~m} / \mathrm{s}$ with an inlet 
temperature of $673.15 \mathrm{~K}$. The wall temperature of the cold side is fixed at $363.15 \mathrm{~K}$. Symmetrical boundaries are defined for the side surfaces of the hot-side flow channel. The voltage at the bottom surface of the rightmost TE leg is assumed to be 0 , and the remaining outer surfaces are assumed to be adiabatic.

\section{Numerical method and data reduction}

As the studied Reynolds number is less than 500, the flow through the hot-side channel is assumed to be three dimensional, laminar, incompressible, and steady. Although there are many vortices in the flow channel with LVGs, several previous experimental and numerical studies have demonstrated that our assumption of laminar flow is valid. Ma et al. [23] conducted an experiment in a rectangular channel with LVGs and showed that the critical Reynolds number for the transition from the laminar to turbulent flow was around 1650. Liu et al. [24] investigated the heat transfer and pressure drop performance of rectangular microchannels with LVGs by experiment, and their work indicated that the critical Reynolds number ranged from 600 to 730 . Tian et al. [25] used a laminar model to simulate a rectangular channel with LVGs. Their numerical results agreed well with the experimental results conducted by Wu and Tao [26] when the Reynolds number was less than 1700.

The continuity, momentum, and energy equations for the fluid domain in the hot-side channel are shown as follows:

$$
\begin{gathered}
\nabla \cdot \mathbf{u}=0 \\
\rho_{\mathrm{f}}(\mathbf{u} \cdot \nabla \mathbf{u})=-\nabla p+\mu_{f} \nabla^{2} \mathbf{u} \\
\rho_{\mathrm{f}} c_{\mathrm{p}, \mathrm{f}} \mathbf{u} \cdot \nabla T=\nabla \cdot\left(k_{\mathrm{f}} \nabla T\right)
\end{gathered}
$$

where $\mathbf{u}$ is the velocity vector, $T$ is the temperature, $p$ is the static pressure. $\rho_{\mathrm{f}}, k_{\mathrm{f}}, \mu_{\mathrm{f}}$ and $c_{\mathrm{p}, \mathrm{f}}$ are the density, thermal conductivity, dynamic viscosity, and heat capacity, respectively, of the exhaust gas.

In the ceramic layers and the hot-side and cold-side plates, only heat conduction is considered. The corresponding heat equation is as follows: 


$$
\nabla \cdot\left(k_{\mathrm{s}} \nabla T\right)=0
$$

where $k_{\mathrm{s}}$ is the thermal conductivity of stainless steel.

In the TE modules and copper layers, the Seebeck, Peltier, Thomson and Joule heating effects are considered in addition to the heat conduction. The corresponding equations are described as follows [27]:

$$
\nabla \cdot\left(-k_{\mathrm{s}} \nabla T+\alpha T \mathbf{J}\right)=\mathbf{J} \cdot \mathbf{E}
$$

where,

$$
\begin{aligned}
& \mathbf{J}=-\sigma(\nabla V+\alpha \nabla T) \\
& \mathbf{E}=-\nabla V
\end{aligned}
$$

here, $k_{s}, \alpha$, and $\sigma$ are the thermal conductivity, Seebeck coefficient, and electrical conductivity of the TE modules or copper layers, respectively. J, E, and $V$ are the electric current density vector, electric field intensity vector, and voltage, respectively.

The electrical current in the TE modules and copper layers follows the conservation law, which is shown as follows:

$$
\nabla \cdot \mathbf{J}=0
$$

It is difficult to simulate all 189 couples of the TE legs in detail. However, the thermal resistance of these TE legs is connected in parallel, while the electrical resistance is in series. We obtain the following Eqns. (9)-(11) according to the heat flux equilibrium between the TE module and TE legs caused by the Peltier effect, heat conduction, and Joule heating.

$$
\begin{gathered}
\alpha_{E} T I=\sum_{i=1}^{N} \alpha_{\operatorname{leg}, i} T I \\
k_{E} A_{E} \frac{\Delta T}{l_{E}}=\sum_{i=1}^{N}\left(k_{\operatorname{leg}, i} A_{\operatorname{leg}, i} \frac{\Delta T}{l_{\operatorname{leg}, i}}\right) \\
I^{2} \rho_{E} \frac{l_{E}}{A_{E}}=\sum_{i=1}^{N}\left(I^{2} \rho_{\operatorname{leg}, i} \frac{l_{\operatorname{leg}, i}}{A_{\operatorname{leg}, i}}\right)
\end{gathered}
$$

Here, $\alpha_{\mathrm{leg}}, k_{\mathrm{leg}}, \rho_{\mathrm{leg}}, l_{\mathrm{leg}}$, and $A_{\mathrm{leg}}$, are the Seebeck coefficient, thermal conductivity, electrical resistivity, length and cross-sectional area for a single TE leg, respectively, 
and $N$ is the number of TE legs. $\alpha_{\mathrm{E}}, k_{\mathrm{E}}, \rho_{\mathrm{E}}, l_{\mathrm{E}}$, and $A_{\mathrm{E}}$ are the equivalent Seebeck coefficient, equivalent thermal conductivity, equivalent electrical resistivity, length and cross-sectional area for a TE module, respectively. It is assumed that all of the thermal-electrical properties of the TE legs are temperature-independent, and all the TE legs are distributed in one stage. Thus the equivalent thermal-electrical properties of the TE module can be derived from those of the TE legs according to the following Eqns. (12)-(14):

$$
\begin{aligned}
& \alpha_{E}=N \alpha_{\text {leg }} \\
& k_{E}=k_{\text {leg }} \\
& \rho_{E}=N^{2} \rho_{\text {leg }}
\end{aligned}
$$

The fluid-thermal-electric multi-physics coupled model is established in the COMSOL $4.4^{\circledR}$ platform by organizing the laminar flow module, heat transfer module, and electric current module. The Seebeck, Peltier, Thomson, and Joule heating effects are considered in the TE modules and copper layers, and the equivalent thermal-electrical properties are used during the numerical simulations. In order to improve the convergence, the laminar flow field is solved by the geometric multigrid iterative solver at first. Then the heat transfer and electric currents fields are solved by the algebraic multigrid iterative solver. Convergence is specified as that the residual for every variable is less than $10^{-4}$.

The Reynolds number $(R e)$ is defined as:

$$
R e=\frac{\rho_{\mathrm{f}} U D_{\mathrm{h}}}{\mu_{\mathrm{f}}}
$$

where $D_{\mathrm{h}}$ is the hydraulic diameter based on the rectangular cross section of the plate fin channel and $U$ is the corresponding inlet velocity. $\rho_{f}$ and $\mu_{f}$ are the density and dynamic viscosity of the exhaust gas, respectively.

The total power output $\left(P_{\mathrm{o}}\right)$ is calculated from:

$$
P_{\mathrm{o}}=\frac{V_{\mathrm{oc}}^{2}}{2\left(R_{\mathrm{In}}+R_{\mathrm{Load}}\right)}
$$


where $V_{\text {oc }}$ is the open circuit voltage, and $R_{\text {In }}$ and $R_{\text {Load }}$ are the internal and external electric resistances, respectively. The maximum total power output is obtained when the internal and external electric resistances are equal. In the following discussion, $P_{\mathrm{o}}$ refers to the maximum power output, i.e., for $R_{\mathrm{In}}=R_{\mathrm{Load}}$.

The net power $\left(W_{\text {net }}\right)$ is defined as the difference between the total power output $\left(P_{\mathrm{o}}\right)$ and pumping power $\left(W_{\Delta p}\right)$, as shown as:

$$
W_{\text {net }}=P_{\mathrm{o}}-W_{\Delta p}
$$

where,

$$
W_{\Delta p}=\dot{V} \Delta l
$$

here, $\dot{V}$ is the volumetric flow rate, and $\Delta p$ is the pressure drop between the inlet and outlet of hot-side channel.

The thermal conversion efficiency $(\eta)$ is described as:

$$
\eta=\frac{W_{\text {net }}}{m c_{\mathrm{p}}\left(T_{\mathrm{h}, \text { in }}-T_{\mathrm{h}, \text { out }}\right)}
$$

where $m, c_{\mathrm{p}}, T_{\mathrm{h}, \text { in }}$, and $T_{\mathrm{h}, \text { out }}$ are the mass flow rate, specific heat, inlet temperature and outlet temperature of the exhaust gas.

\section{Grid independence test and code validation}

The grid system has a significant effect on the numerical results, especially on the calculation of the flow field. Here, five different grid systems, with element ranges from 0.2 million to 4 million, are tested. The results show that the relative errors are less than $3 \%$ when the element number is greater than 1.14 million compared to the grid system with 4 million elements. Therefore, the grid system with 1.14 million is selected for the following calculations considering the computer source, calculation time and calculation precision. Structured elements are used for the hot-side channel and TE modules, while unstructured elements are used for the remaining domains.

In our previous study [22], the fluid-thermal-electric multi-physics coupled model for the TEG was validated with the results of Reddy et al. [28], which showed that the maximum relative deviations were less than $6 \%$. However, in that prior 
validation the detailed TE legs were simulated. Therefore, the present method of using equivalent physical properties for the TE module should be further validated before proceeding. Figure 3 shows a comparison of the present study with the experimental data in Ref. [29]. The numerical geometry and boundary conditions are the same as those in the experiment [29]. The dimensions of the TE module are $30 \mathrm{~mm} \times 30 \mathrm{~mm} \times$ $3.42 \mathrm{~mm}$. One TE module is modeled, which includes 31 pairs of p-type or n-type TE legs. The spacing of the TE module is $200 \mathrm{~mm}$ and the spreader thickness is $5 \mathrm{~mm}$. The cold-side fluid of the TE module is cold water, with a temperature of $300 \mathrm{~K}$ and a heat transfer coefficient of $1000 \mathrm{~W} /\left(\mathrm{m}^{2} \cdot \mathrm{K}\right)$. The hot-side fluid of the TE module is the flue gas, with a heat transfer coefficient of $20 \mathrm{~W} /\left(\mathrm{m}^{2} \cdot \mathrm{K}\right)$. Different hot-side temperatures of $400 \mathrm{~K}$ and $500 \mathrm{~K}$ are studied. The averaged relative errors between the present study and the experimental results in Ref. [29] are $9.5 \%$ for $\Delta T=200 \mathrm{~K}$ and $15.9 \%$ for $\Delta T=100 \mathrm{~K}$. This reasonable good agreement validates the present numerical method.

In order to validate whether the present numerical method is valid for the heat exchanger with LVGs, a rectangular channel with LVGs shown in Refs. [25] and [26] is modeled. The dimensions of the channel are $400 \mathrm{~mm}$ (length) $\times 160 \mathrm{~mm}$ (width) $\times$ $27 \mathrm{~mm}$ (height). The LVGs are delta-winglet shaped, which have a chord length of 60 $\mathrm{mm}$, a height of $27 \mathrm{~mm}$, and an inclined angle of $30^{\circ}$. Inlet velocities ranging from $0.25 \mathrm{~m} / \mathrm{s}$ to $0.97 \mathrm{~m} / \mathrm{s}$, with corresponding Reynolds numbers from 475 to 1855 are studied. The results are compared with the experimental results in Ref. [26] and numerical results in Ref. [25], as shown in Fig. 4. It can be seen that the present results agree well with the numerical results in Ref. [25] and experimental results in Ref. [26]. The maximum relative error is $9 \%$ compared with the experimental results in Ref. [26]. It should be noted that both the present study and the numerical study in Ref. [25] use a laminar model to conduct the numerical simulation. Therefore, the laminar model is valid for the rectangular channel with LVGs for Reynolds numbers examined in this work.

\section{Results and discussion}




\subsection{Effect of LVGs on the performance of the TEG}

Figure 5(a) shows the velocity and voltage distributions of the TEGs with a smooth channel under the baseline operating conditions. Here, the inlet velocity of the hot-side exhaust gas in the plate fin channel is $2 \mathrm{~m} / \mathrm{s}$ and the corresponding Reynolds number is 389 . The inlet temperature of the hot-side exhaust gas is $673.15 \mathrm{~K}$, while the wall temperature of the cold side is $363.15 \mathrm{~K}$. Because there are no ribs in the smooth channel, a thick flow boundary layer forms on the wall surface of the hot-side channel, which results in the formation of a thermal boundary layer. The streamlines shown in Fig. 5(b) support the explanation. Almost all of the streamlines are parallel to the flow direction indicating how the flow boundary layer is formed. The maximum velocity in the hot-side channel is $3.53 \mathrm{~m} / \mathrm{s}$, and, as expected, the voltage becomes larger and larger from the rightmost n-type leg to the leftmost p-type leg. The maximum generated open circuit voltage is $2.26 \mathrm{~V}$. The voltage generated by the three TE modules becomes less and less from the leftmost TE module to the rightmost TE module. This phenomenon is explained later in the discussion.

When the LVGs are attached to the smooth channel, the fluid flow is disturbed and vortices are formed downstream from every LVG, as shown in Fig. 6(a). The thickness of flow boundary layer is much less than that of the TEG with smooth channel. Figure 6(b) shows the LVG disturbance in detail. Strong three-dimensional vortices are observed in the downstream of LVGs, and the streamlines are no longer straight due to the vortices. The maximum local velocity also increases due to the small cross section around the LVGs. Compared to the flow in a smooth channel shown in Fig. 5(a), the maximum velocity increases from $3.53 \mathrm{~m} / \mathrm{s}$ to $5.46 \mathrm{~m} / \mathrm{s}$. The flow interruption results in an enhancement in the heat transfer, and thus a corresponding increase in the voltage generation. The maximum open circuit voltage increases from $2.26 \mathrm{~V}$ to $3.5 \mathrm{~V}$. Similarly, the voltage generated by the three TE modules becomes less and less from the leftmost TE module to the rightmost TE module.

Figure 7 shows the comparison of the temperature difference in each TE module under the baseline operating conditions, and it is clear that the temperature difference 
in each module decreases along the flow direction for both the smooth channel and the LVG channel. The reason is that the temperature on the hot-side channel decreases as the hot exhaust gas is cooled, but the temperature on the cold-side channel remains unchanged. The temperature differences in the LVG channel are increased by $41 \%-75 \%$ when compared with the smooth channel due to the strong disturbance by the LVGs as shown in Fig. 6(b).

The comparison of the open circuit voltage in each TE module under the baseline condition is shown in Fig. 8. As expected, the voltage generated by each module decreases both in the smooth channel and in the LVG channel due to the fact that the voltage generated by the Seebeck effect is proportional to the temperature difference between the hot and cold sides of the TE modules. Thus, the voltage distribution follows a similar trend as the temperature difference shown in Fig. 7. However, the voltage generated by the TEG with LVGs in the channel is increased by $41 \%-75 \%$ when compared to the voltage generated by the TEG with the smooth channel.

\subsection{Effect of the Reynolds number on the performance of the TEG}

The velocity typically has a significant effect on the thermal-hydraulic performance of a heat exchanger. The effect of the Reynolds number on the heat input and pressure drop performance of the TEG is examined and shown in Fig. 9. Here, the inlet temperature of hot-side exhaust gas is fixed at $673.15 \mathrm{~K}$, while the wall temperature of the cold side is fixed at $363.15 \mathrm{~K}$. These results show that the heat input in the smooth channel and in the LVG channel increases with increasing Reynolds number, although the rate of increase begins to diminish at higher Reynolds number. The pressure drop in the smooth channel and in the LVG channel also increases rapidly with increasing Reynolds number. The LVG channel produces a larger pressure drop that continues to climb significantly at high Reynolds number. Overall, including LVGs in the channel leads to an increase in the heat input of $26 \%-63 \%$, with a corresponding increase in the pressure drop of $131 \%-368 \%$ for the range of Reynolds numbers examined in this work.

The purpose of increasing the heat input improves the Seebeck voltage and thus 
improving the power output. As shown in Fig. 10, the total power output in the TEG with the LVG channel is much greater than the power generated in the TEG with the smooth channel, especially at larger Reynolds numbers. Compared to the smooth channel, the total power output is increased by $59 \%-153 \%$ by the LVGs, which is a greater percentage increase than the increase of the heat input. The physical reason for this behavior is that the power output is proportional to the square of voltage, while the Seebeck voltage is simply proportional to the temperature difference. Thus even modest improvements in heat input lead to larger gains in power output.

The additional pressure drop produced by the LVGs has a negative effect on the net power output because the increase in pressure drop requires additional pumping power. However, in this work, all of the ratios of pumping power to total power output are less than $2 \%$, which could be neglected at $T_{\mathrm{h}, \mathrm{in}}=673.15 \mathrm{~K}$ and $T_{\mathrm{c}}=363.15 \mathrm{~K}$. Therefore, the net power exhibits a similar trend as the total power output, as shown in Fig. 11. The net power in the LVG channel is $59 \%-150 \%$ greater than for the smooth channel.

As the Reynolds number increases, the thermal efficiency in both the smooth channel and LVG channel increases, as also shown in Fig. 11. The thermal efficiency increases if the heat input increases and the pumping power remains small. Therefore, enhancing the heat transfer performance is an effective way to enhance the comprehensive performance of TEG, provided that the increase in heat input does not require significant increases in pumping power. The thermal efficiency in the LVG channel is increased by $26 \%$ to $58 \%$ compared to the case with the smooth channel for Reynolds numbers between 97 and 487. The maximum net power is $0.6 \mathrm{~W}$ and the maximum thermal efficiency is $1.5 \%$ in the $\mathrm{LVG}$ channel at $T_{\mathrm{h}, \mathrm{n}}=673.15 \mathrm{~K}$ and $T_{\mathrm{c}}=363.15 \mathrm{~K}$.

\subsection{Effect of the hot-side inlet temperature on the performance of the TEG}

The effect of the hot-side inlet temperature on the heat input and open circuit voltage at $R e=487$ and $T_{\mathrm{c}}=363.15 \mathrm{~K}$ is described in Fig. 12. The results demonstrate that the heat input and open circuit voltage increase linearly with increasing hot-side 
inlet temperature. For the same Reynolds number, the heat input and open circuit voltage in the LVG channel are 55\% higher than in the smooth channel. It should be noted that all the physical properties are assumed to be constant so that the increment ratios at different Reynolds numbers are the same. If temperature-dependent physical properties are considered, it may result in some differences in the increment ratios. For the LVG and smooth channels, the heat input and open circuit voltage for an inlet temperature of $673.15 \mathrm{~K}$ are increased by $182 \%$ compared to those properties for an inlet temperature of $473.15 \mathrm{~K}$.

The effect of hot-side inlet temperature on the total power output is plotted in Fig. 13. As described previously, the total power output is proportional to the square of the open circuit voltage, while the Seebeck voltage is proportional to the temperature difference. Moreover, the open circuit voltage increases linearly with the increase of the hot-side inlet temperature. Therefore, the curve of the total power output as a function of the hot-side inlet temperature shows a parabolic-like trend. The total power output in the LVG channel is $141 \%$ higher than for the smooth channel at different hot-side inlet temperatures. For the LVG and smooth channels, the total power output is improved by a factor of 6.9 when the hot-side inlet temperature increases from $473.15 \mathrm{~K}$ to $673.15 \mathrm{~K}$. Therefore, the ratio of pumping power to power output decreases rapidly with the increase of the hot-side inlet temperature. The ratio of pumping power to power output in the LVG channel is $9.9 \%$ at $473.15 \mathrm{~K}$, but only $1.2 \%$ at $673.15 \mathrm{~K}$. However, the ratio of pumping power to power output in the smooth channel is much smaller, at $5.8 \%$ for $473.15 \mathrm{~K}$ and $0.7 \%$ for $673.15 \mathrm{~K}$.

As shown in Fig. 14, the net power and thermal conversion efficiency both in the smooth channel and LVG channel increase with an increase of the hot-side inlet temperature. For the LVG channel, the net power is $0.05 \mathrm{~W}$ at $473.15 \mathrm{~K}$ and $0.48 \mathrm{~W}$ at $673.15 \mathrm{~K}$, while the thermal conversion efficiency is $0.45 \%$ at $473.15 \mathrm{~K}$ and $1.4 \%$ at 673.15 K. Again, the net power and thermal conversion efficiency in the LVG channel are much greater than those in the smooth channel. However, the pressure drop has a negative effect on the net power and thermal efficiency, which is more obvious in a channel with large pressure drop such as the LVG channel. Compared to the smooth 
channel, the net power in the LVG channel is improved by $130 \%$ at $473.15 \mathrm{~K}$ and $140 \%$ at $673.15 \mathrm{~K}$, while the thermal conversion efficiency is increased by $49 \%$ at $473.15 \mathrm{~K}$ and $55 \%$ at $673.15 \mathrm{~K}$.

\subsection{Effect of the cold-side temperature on the performance of the TEG}

In this section, the effect of the cold-side temperature on the performance of the TEG at $R e=487$ and $T_{\mathrm{h}, \mathrm{in}}=673.15 \mathrm{~K}$ is studied. As shown in Fig. 15, both the heat input and open circuit voltage decrease linearly with an increase of the cold-side temperature. For both the smooth channel and LVG channel, the heat input and open circuit voltage are improved by $12.9 \%$ when the cold-side temperature decreases from $363.15 \mathrm{~K}$ to $323.15 \mathrm{~K}$. When compared carefully with Fig. 12, it can be seen that the performance enhancement produced by reducing the cold-side temperature is smaller than the gains generated by increasing the hot-side temperature. The reason may rely on the fact that the thermal resistance on the hot side is much greater than that on the cold side.

As described in Fig. 16, the total power output in both the smooth channel and LVG channel decreases with an increase of the cold-side temperature. Therefore, the ratio of pumping power to power output increases as the cold-side temperature increases. For both channels, the total power output is enhanced by $27.4 \%$ when the cold-side temperature decreases from $363.15 \mathrm{~K}$ to $323.15 \mathrm{~K}$. The maximum ratio of pumping power to power output is only $1.24 \%$. Due to the small pressure drop, the net power exhibits almost the same trend as the total power output, as shown in Fig. 17. The maximum net power in the LVG channel is $0.62 \mathrm{~W}$ at $323.15 \mathrm{~K}$. The thermal conversion efficiency in both channels decreases as the cold-side temperature increases. Compared to the smooth channel, the thermal efficiency in LVG channel is enhanced by $55 \%$. The maximum thermal conversion efficiency for the LVG channel is $1.02 \%$ at $323.15 \mathrm{~K}$.

\subsection{Effect of the cold-side thermal boundary on the performance of the TEG}

In a vehicle, the cooling water for the engine can be used as the cold-side fluid of 
the TEG. The coolant temperature is generally between $80^{\circ} \mathrm{C}$ and $90^{\circ} \mathrm{C}$, and its convective heat transfer coefficient is around $1000 \mathrm{~W} /\left(\mathrm{m}^{2} \cdot \mathrm{K}\right)^{[30]}$. The effects of the cold-side thermal boundary, i.e., constant temperature boundary or constant heat transfer coefficient on the heat input and voltage of the smooth channel and the LVG channel are shown in Figs. 18 and 19. Here, the heat transfer coefficient of the cold-side cooling water is specified as $1000 \mathrm{~W} /\left(\mathrm{m}^{2} \cdot \mathrm{K}\right)$. It can be seen that there are few differences between the two kinds of thermal boundary since the convective heat transfer coefficient is so large for the liquid coolant. Therefore, the cold-side temperature of the TEG is very close to the temperature of the cooling water, even when a constant heat transfer coefficient is specified at the cold side of the TEG. The differences of heat input and open circuit voltage in the smooth channel are less than $3.5 \%$, while they are less than $5.4 \%$ in the LVG channel. These results indicate that the differences between the two kinds of thermal boundary become slightly larger when the heat transfer performance on the hot side is enhanced. However, this deviation remains small and acceptable for this study.

\subsection{Comparison of the performance between the LVG and the smooth channels used for waste heat recovery in vehicles}

In order to evaluate the potential of LVGs used for actual waste heat recovery in vehicles, some typical working conditions for the vehicle engine are studied. The temperature of the cooling water is assumed to be $90^{\circ} \mathrm{C}$, and its convective heat transfer coefficient is $1000 \mathrm{~W} /\left(\mathrm{m}^{2} \cdot \mathrm{K}\right)$, while the hot-side inlet temperature is taken as $500^{\circ} \mathrm{C}^{[30]}$. In order to obtain more accurate results, the temperature-dependent physical properties of TE materials are now used. These values are given in Angrist's book $^{[31]}$, and the corresponding fitted polynomials were proposed by Reddy et al. ${ }^{[32]}$.

The polynomials for the Seebeck coefficient, electrical resistivity, and thermal conductivity of n-type TE leg are shown in Eqns. (20)-(22), respectively.

$$
\begin{gathered}
\alpha=-10^{-6} \times\left[151.7414+0.226 T+1.574 \times 10^{-3} T^{2}-1.3005 \times 10^{-5} T^{3}\right. \\
\left.+2.54021 \times 10^{-8} T^{4}-1.6143 \times 10^{-11} T^{5}\right], \quad \forall 25^{\circ} \mathrm{C} \leq T \leq 550^{\circ} \mathrm{C} \\
\rho=10^{-5} \times\left[0.93562+4.321 \times 10^{-3} T-7.6044 \times 10^{-5} T^{2}+7.2921 \times 10^{-7} T^{3}-2.7176\right. \\
\left.\times 10^{-9} T^{4}+4.3202 \times 10^{-12} T^{5}-2.49 \times 10^{-15} T^{6}\right], \forall 25^{\circ} \mathrm{C} \leq T \leq 550^{\circ} \mathrm{C}
\end{gathered}
$$




$$
k=\left\{\begin{array}{rr}
1.2979-4.3139 \times 10^{-3} T+8.8823 \times 10^{-5} T^{2}-4.2988 \times 10^{-7} T^{3}, \\
\forall 25^{\circ} \mathrm{C} \leq T \leq 100^{\circ} \mathrm{C} \\
0.81325+1.0936 \times 10^{-2} T-7.454 \times 10^{-5} T^{2}+1.8004 \times 10^{-7} T^{3} \\
-1.3833 \times 10^{-10} T^{4}, & \forall 100^{\circ} \mathrm{C}<T \leq 400^{\circ} \mathrm{C} \\
-4376.63+47.82405 T-0.2087 T^{2}+4.548094 \times 10^{-4} T^{3} \\
-4.950144 \times 10^{-7} T^{4}+2.15322 \times 10^{-10} T^{5}, & \forall 400^{\circ} \mathrm{C}<T \leq 550^{\circ} \mathrm{C}
\end{array}\right.
$$

The polynomials for the Seebeck coefficient, electrical resistivity and thermal conductivity of p-type TE leg are shown in Eqns. (23)-(25), respectively.

$$
\begin{aligned}
& \alpha=\left\{\begin{array}{lr}
10^{-6} \times\left[208.4305-0.72663 T+2.3556 \times 10^{-2} T^{2}-3.265 \times 10^{-4} T^{3}\right. \\
\left.+2.2189 \times 10^{-6} T^{4}-5.6125 \times 10^{-9} T^{5}\right], & \forall 20^{\circ} \mathrm{C} \leq T \leq 170^{\circ} \mathrm{C} \\
10^{-6} \times\left[512.3379-2.4027 T+4.8969 \times 10^{-3} T^{2}-4.13146 \times 10^{-6} T^{3}\right], \\
& \forall 170^{\circ} \mathrm{C}<T \leq 450^{\circ} \mathrm{C}
\end{array}\right. \\
& \rho=10^{-5} \times\left[1.14586-7.9785 \times 10^{-3} T+1.69084 \times 10^{-4} T^{2}-7.526 \times 10^{-7} T^{3}\right. \\
& \left.+1.33902 \times 10^{-9} T^{4}-8.9007 \times 10^{-13} T^{5}\right], \quad \forall 20^{\circ} \mathrm{C} \leq T \leq 450^{\circ} \mathrm{C} \\
& k=\left\{\begin{array}{lc}
0.874746+8.085 \times 10^{-3} T-3.63173 \times 10^{-5} T^{2}-1.16321 \times 10^{-6} T^{3} \\
+1.345 \times 10^{-8} T^{4}-4.0425 \times 10^{-11} T^{5}, & \forall 20^{\circ} \mathrm{C} \leq T \leq 170^{\circ} \mathrm{C} \\
1.84097-4.511 \times 10^{-3} T-1.415 \times 10^{-5} T^{2}+7.033 \times 10^{-8} T^{3}, \\
\forall 170^{\circ} \mathrm{C}<T \leq 370^{\circ} \mathrm{C} \\
-234.94675+1.6305 T-3.69338 \times 10^{-3} T^{2}+2.74356 \times 10^{-6} T^{3}, \\
\forall 370^{\circ} \mathrm{C}<T \leq 450^{\circ} \mathrm{C}
\end{array}\right.
\end{aligned}
$$

The temperature-dependent physical properties of copper are also considered. The polynomials for the electrical resistivity and thermal conductivity of copper are shown in Eqns. (26) and (27) ${ }^{[32]}$.

$$
\begin{aligned}
& \rho=10^{-8} \times\left[1.5113+7.122 \times 10^{-3} \mathrm{~T}\right], \quad \forall 25^{\circ} \mathrm{C} \leq T \leq 650^{\circ} \mathrm{C} \\
& k=402.104-6.874 \times 10^{-2} \mathrm{~T}, \quad \forall 25^{\circ} \mathrm{C} \leq T \leq 800^{\circ} \mathrm{C}
\end{aligned}
$$

As demonstrated in the above polynomials, the physical properties of TE materials are sensitive to the temperature. Moreover, the physical properties of TE materials change non-linearly with the variation of temperature. A comparison of performance between the LVG and the smooth channels used for waste heat recovery in vehicles under the typical working conditions are shown in Table 2. Once again, these results demonstrate that the LVGs have significant potential to enhance the 
performance of the TEGs in vehicles. Compared to the traditional plain plate-fin heat exchanger, the heat input, open circuit voltage, and thermal conversion efficiency in the TEG with the LVGs are increased by $49.4 \%, 52.1 \%$ and $54.5 \%$, respectively. Moreover, the increased pumping power caused by the LVGs has little effect on the net power output.

\section{Conclusions}

Plate-fin heat exchangers are widely used in large-scale TEGs as a heat sink. In this paper, a fluid-thermal-electric multi-physics coupled model for the gas-to-liquid TEG is established on the COMSOL $4.4{ }^{\circledR}$ platform by organizing the laminar flow module, heat transfer module, and electric currents module. The Seebeck, Peltier, and Thomson effects along with Joule heating are considered in the TE modules. In order to simplify the numerical model of the large-scale TEG, the equivalent thermal-electrical properties of the TE module are used to replace the specific thermal-electrical properties of the TE legs. This numerical method is validated by comparing results with experimental data in the open literature. The main conclusions from this work are summarized as follows:

(1) Comparisons of velocity, voltage and streamline distributions indicate that the LVGs could produce complex three-dimensional vortices in the cross section downstream from the LVGs, thus enhancing the heat transfer performance and electric performance of the TEG. Under the baseline operating condition, the heat input and open circuit voltage of the TEG with LVGs in the channel are increased by $41 \%-75 \%$ compared to a TEG with a smooth channel. The temperature difference and generated voltage in each module becomes smaller along the flow direction.

(2) Compared to the smooth channel, the heat input and pressure drop in the LVG channel are much larger than those in the smooth channel, especially at higher Reynolds numbers. The heat input is enhanced by $26 \%-63 \%$ while the pressure drop is increased by $131 \%-368 \%$. However, the pressure drop has little effect on the comprehensive performance of TEG in the studied cases since the pumping power is small compared to the power generated. The total power output is improved by 
$59 \%-153 \%$ and the thermal efficiency is improved by $26 \%-58 \%$. The maximum net power is $0.6 \mathrm{~W}$ and the maximum thermal efficiency is $1.5 \%$ in the LVG channel at $T_{\mathrm{h}, \mathrm{in}}=673.15 \mathrm{~K}, T_{\mathrm{c}}=363.15 \mathrm{~K}$ and $R e=487$.

(3) The hot-side inlet temperature has a significant effect on the performance of the TEG. The heat input and open circuit voltage increase rapidly as the hot-side inlet temperature increases. For the LVG and smooth channels, the heat input and open circuit voltage at $673.15 \mathrm{~K}$ are increased by $182 \%$ compared to those at $473.15 \mathrm{~K}$. However, the cold-side temperature has little effect on the performance of the TEG compared to the hot-side inlet temperature. For both the smooth channel and the LVG channel, the heat input and open circuit voltage are improved by $12.9 \%$ when the cold-side temperature decreases from $363.15 \mathrm{~K}$ to $323.15 \mathrm{~K}$.

(4) The performances of the TEGs are almost the same whether a constant temperature boundary or a constant heat transfer coefficient boundary are applied to the cold-side of the TEG. An example based on the working conditions of an actual vehicle engine and that includes the temperature-dependent physical properties of TE materials demonstrates that the LVGs have significant potential to enhance the performance of TEG.

\section{Acknowledgements}

This material is based upon work supported by the National Natural Science Foundation of China (Grant No. 51306139), International Cooperation and Exchanges Project of NSFC of China (Grant No.51120165002), and the U.S. National Science Foundation and Department of Energy through the NSF/DOE Joint Thermoelectric Partnership (Grant No. CBET-1048708).

\section{References}

[1] Bell LE. Cooling, heating, generating power, and recovering waste heat with thermoelectric systems. Science 2008; 321(895): 1457-1461.

[2] Poudel B, Hao Q, Ma Y, Lan YC, Minnich A, Yu B, Yan XA, Wang DZ, Muto A, Vashaee D, Chen XY, Liu JM, Dresselhaus MS, Chen G, Ren ZF. High-thermoelectric 
performance of nanostructured bismuth antimony telluride bulk alloys. Science 2008; 320(5876): 634-638.

[3] Kraemer D, Poudel B, Feng HP, Caylor JC, Yu B, Yan X, Ma Y, Wang XW, Wang DZ, Muto A, McEnaney K, Chiesa M, Ren ZF, Chen G. High-performance flat-panel solar thermoelectric generators with high thermal concentration. Nature Materials 2011; 10(7): 532-538.

[4] Hsiao YY, Chang WC, Chen SL. A mathematic model of thermoelectric module with applications on waste heat recovery from automobile engine. Energy 2010; 35(3): $1447-1454$

[5] In BD, Kim HI, Son JW, Lee KH. The study of a thermoelectric generator with various thermal conditions of exhaust gas from a diesel engine. International Journal of Heat and Mass Transfer 2015; 86: 667-680.

[6] Liu X, Deng YD, Li Z, Su CQ. Performance analysis of a waste heat recovery thermoelectric generation system for automotive application. Energy Conversion and Management 2015; 90: 121-127.

[7] Favarel C, Bédécarrats JP, Kousksou T, Champier D. Numerical optimization of the occupancy rate of thermoelectric generators to produce the highest electrical power. Energy 2014; 68: 104-116.

[8] Liang XY, Sun XX, Tian H, Shu GQ, Wang YS, Wang X. Comparison and parameter optimization of a two-stage thermoelectric generator using high temperature exhaust of internal combustion engine. Applied Energy 2014; 130: 190-199.

[9] Yu SH, Du Q, Diao H, Shu GQ, Jiao K. Start-up modes of thermoelectric generator based on vehicle exhaust waste heat recovery. Applied Energy 2015; 138: 276-290.

[10] Chen WH, Huang SR, Lin YL. Performance analysis and optimum operation of a thermoelectric generator by Taguchi method. Applied Energy 2015; 158: 44-54.

[11] Crane DT, Jackson GS. Optimization of cross flow heat exchangers for thermoelectric waste heat recovery. Energy Conversion and Management 2004; 45 (9-10): 1565-1582. 
[12] Lu HL, Wu T, Bai SQ, Xu KC, Huang YJ, Gao WM, Yin XL, Chen LD. Experiment on thermal uniformity and pressure drop of exhaust heat exchanger for automotive thermoelectric generator. Energy 2013; 54: 372-377.

[13] Zhou SY, Sammakia BG, White B, Borgesen P. Multiscale modeling of thermoelectric generators for the optimized conversion performance. International Journal of Heat and Mass Transfer 2013; 62: 435-444.

[14] Lesage FJ, Sempels ÉV, Lalande-Bertrand N. A study on heat transfer enhancement using flow channel inserts for thermoelectric power generation. Energy Conversion and Management 2013; 75: 532-541.

[15] Amaral C, Brandão C, Sempels ÉV, Lesage FJ. Net thermoelectric generator power output using inner channel geometries with alternating flow impeding panels. Applied Thermal Engineering 2014; 65: 94-101.

[16] Reddy BVK, Barry M, Li J, Chyu MK. Enhancement of thermoelectric device performance through integrated flow channels. Frontiers in Heat and Mass Transfer 2013; 4: 023001 .

[17] Wang TC, Luan WL, Wang W, Tu ST. Waste heat recovery through plate heat exchanger based thermoelectric generator system. Applied Energy 2014; 136: 860-865.

[18] Pandit J, Thompson M, Ekkad SV, Huxtable ST. Effect of pin fin to channel height ratio and pin fin geometry on heat transfer performance for flow in rectangular channels. International Journal of Heat and Mass Transfer 2014; 77: 359-368.

[19] Liu C, Teng JT, Chu JC. Experimental investigations on liquid flow and heat transfer in rectangular microchannel with longitudinal vortex generators. International Journal of Heat and Mass Transfer 2011; 54(13-14): 3069-3080.

[20] Wang QW, Chen QY, Wang L, Zeng M, Huang YP, Xiao ZJ. Experimental study of heat transfer enhancement in narrow rectangular channel with longitudinal vortex generators. Nuclear Engineering and Design 2007; 237(7): 686-693.

[21] He J, Liu L, Jacobi AM. Air-Side Heat-Transfer Enhancement by a New Winglet-Type Vortex Generator Array in a Plain-Fin Round-Tube Heat Exchanger. ASME Journal of Heat Transfer 2010; 132(7): 071801. 
[22] Ma T, Pandit J, Ekkad SV, Huxtable ST, Wang QW. Simulation of thermoelectric-hydraulic performance of a thermoelectric power generator with longitudinal vortex generators. Energy 2015; 84: 695-703.

[23] Ma J, Huang YP, Huang J, Wang YL, Wang QW. Experimental investigations on single-phase heat transfer enhancement with longitudinal vortices in narrow rectangular channel. Nuclear Engineering and Design 2010; 240: 92-102.

[24] Liu C, Teng J, Chu J, Chiu Y, Huang S, Jin S, Dang T, Greif R, Pan H. Experimental investigations on liquid flow and heat transfer in rectangular microchannel with longitudinal vortex generators. International Journal of Heat and Mass Transfer 2011; 54: 3069-3080.

[25] Tian LT, He YL, Lei YG, Tao WQ. Numerical study of fluid flow and heat transfer in a flat-plate channel with longitudinal vortex generators by applying field synergy principle analysis. International Communications in Heat and Mass Transfer 2009; 36: 111-120.

[26] Wu JM, Tao WQ. Numerical study on laminar convection heat transfer in a channel with longitudinal vortex generator. Part B: Parametric study of major influence factors. International Journal of Heat and Mass Transfer 2008; 51: 3683-3692.

[27] COMSOL Multiphysics. COMSOL solver theory guide 4.4. Burlington, MA, USA: COMSOL Inc.;2014

[28] Reddy BVK, Barry M, Li J, Chyu MK. Three Dimensional Multiphysics Coupled Field Analysis of an Integrated Thermoelectric Device. Numerical Heat Transfer, Part A: Applications: An International Journal of Computation and Methodology 2012; 62: 933-947.

[29] Jang JY, Tsai YC. Optimization of thermoelectric generator module spacing and spreader thickness used in a waste heat recovery system. Applied Thermal Engineering 2013; 51: 677-689.

[30] Wang Y, Dai C, Wang S. Theoretical analysis of a thermoelectric generator using exhaust gas of vehicles as heat source. Applied Energy 2013; 112: 1171-1180.

[31] Angrist SW. Direct Energy Conversion, 3rd Edition, Boston, USA: Allyn and 
Bacon Inc.; 1976.

[32] Reddy BVK, Barry M, Li J, Chyu MK. Three Dimensional Multiphysics Coupled Field Analysis of an Integrated Thermoelectric Device. Numerical Heat Transfer, Part A: Applications: An International Journal of Computation and Methodology 2012;62(12):933-947. 


\section{Table captions}

Table 1 Physical properties of TEG materials ${ }^{[29]}$.

Table 2 Comparison of the performance between LVG and smooth channels used for waste heat recovery in vehicles.

\section{Figure captions}

Fig. 1. Physical model of the baseline TEG with a smooth channel.

Fig. 2. Physical model of the TEG with LVGs placed in the channel.

Fig. 3. Validation of the method of using equivalent physical properties for the TE module.

Fig. 4. Validation of the heat transfer performance in a rectangular channel with LVGs.

Fig. 5. Velocity, voltage, and streamlines of the TEG with a smooth channel under baseline operating conditions. (a) Velocity and voltage distributions of the TEG with a smooth channel, (b) Streamlines of the TEG with a smooth channel.

Fig. 6. Velocity, voltage, and streamlines of the TEG with LVGs in the channel under baseline operating conditions. (a) Velocity and voltage distributions of the TEG with LVGs in the channel. (b) Streamlines of the TEG with LVGs in the channel.

Fig. 7. Comparison of the temperature difference in each TE module under baseline operating conditions.

Fig. 8. Comparison of the open circuit voltage in each TE module under baseline operating conditions.

Fig. 9. Effect of the Reynolds number on the heat input and pressure drop at $T_{\mathrm{h}, \mathrm{in}}=673.15 \mathrm{~K}$ and $T_{\mathrm{c}}=363.15 \mathrm{~K}$.

Fig. 10. Effect of the Reynolds number on the total power output and the ratio of pumping power to total power output at $T_{\mathrm{h}, \mathrm{in}}=673.15 \mathrm{~K}$ and $T_{\mathrm{c}}=363.15 \mathrm{~K}$.

Fig. 11. Effect of the Reynolds number on the net power and thermal conversion 
efficiency at $T_{\mathrm{h}, \mathrm{in}}=673.15 \mathrm{~K}$ and $T_{\mathrm{c}}=363.15 \mathrm{~K}$.

Fig. 12. Effect of the hot-side inlet temperature on the heat input and open circuit voltage at $R e=487$ and $T_{\mathrm{c}}=363.15 \mathrm{~K}$.

Fig. 13. Effect of the hot-side inlet temperature on the total power output and the ratio of pumping power to total power output at $R e=487$ and $T_{\mathrm{c}}=363.15 \mathrm{~K}$.

Fig. 14. Effect of the hot-side inlet temperature on the net power and thermal conversion efficiency at $R e=487$ and $T_{\mathrm{c}}=363.15 \mathrm{~K}$.

Fig. 15. Effect of the cold-side temperature on the heat input and open circuit voltage at $R e=487$ and $T_{\mathrm{h}, \mathrm{in}}=673.15 \mathrm{~K}$.

Fig. 16. Effect of the cold-side inlet temperature on the total power output and the ratio of pumping power to total power output at $R e=487$ and $T_{\mathrm{h}, \mathrm{in}}=673.15 \mathrm{~K}$.

Fig. 17. Effect of the cold-side inlet temperature on the net power and thermal conversion efficiency at $R e=487$ and $T_{\mathrm{h}, \mathrm{in}}=673.15 \mathrm{~K}$.

Fig. 18. Effect of the cold-side thermal boundary on the heat input and voltage of the TEG with the smooth channel.

Fig. 19. Effect of the cold-side thermal boundary on the heat input and voltage of the TEG with LVGs in the channel. 
Table 1 Physical properties of TEG materials ${ }^{[29]}$.

\begin{tabular}{lll}
\hline \hline Components & Parameters & Value \\
\hline \multirow{2}{*}{ TE leg } & Thermal conductivity, $k$ & $1.6 \mathrm{~W} /(\mathrm{m} \cdot \mathrm{K})$ \\
& Seebeck coefficient, $\alpha$ & $\pm 2.0 \times 10^{-4} \mathrm{~V} / \mathrm{K}$ \\
& Electrical resistivity, $\rho$ & $9.0 \times 10^{-6} \Omega \cdot \mathrm{m}$ \\
\hline \multirow{2}{*}{ Copper connector } & Thermal conductivity, $k$ & $403.0 \mathrm{~W} /(\mathrm{m} \cdot \mathrm{K})$ \\
& Seebeck coefficient, $\alpha$ & $1.4 \times 10^{-5} \mathrm{~V} / \mathrm{K}$ \\
\hline Ceramic layer & Electrical resistivity, $\rho$ & $1.44 \times 10^{-8} \Omega \cdot \mathrm{m}$ \\
\hline \hline
\end{tabular}


Table 2 Comparison of the performance between LVG and smooth channels used for waste heat recovery in vehicles.

\begin{tabular}{lllllll}
\hline \hline Channel & $Q_{\mathrm{h}}(\mathrm{W})$ & $\Delta p(\mathrm{~Pa})$ & $V_{\text {oc }}(\mathrm{V})$ & $W_{\Delta p}(\mathrm{~W})$ & $W_{\text {net }}(\mathrm{W})$ & $\eta$ \\
\hline Smooth & 27.59 & 3.62 & 3.82 & $1.45 \times 10^{-3}$ & 0.58 & $2.09 \%$ \\
LVG & 41.23 & 15.13 & 5.81 & $6.05 \times 10^{-3}$ & 1.33 & $3.23 \%$ \\
\hline \hline
\end{tabular}




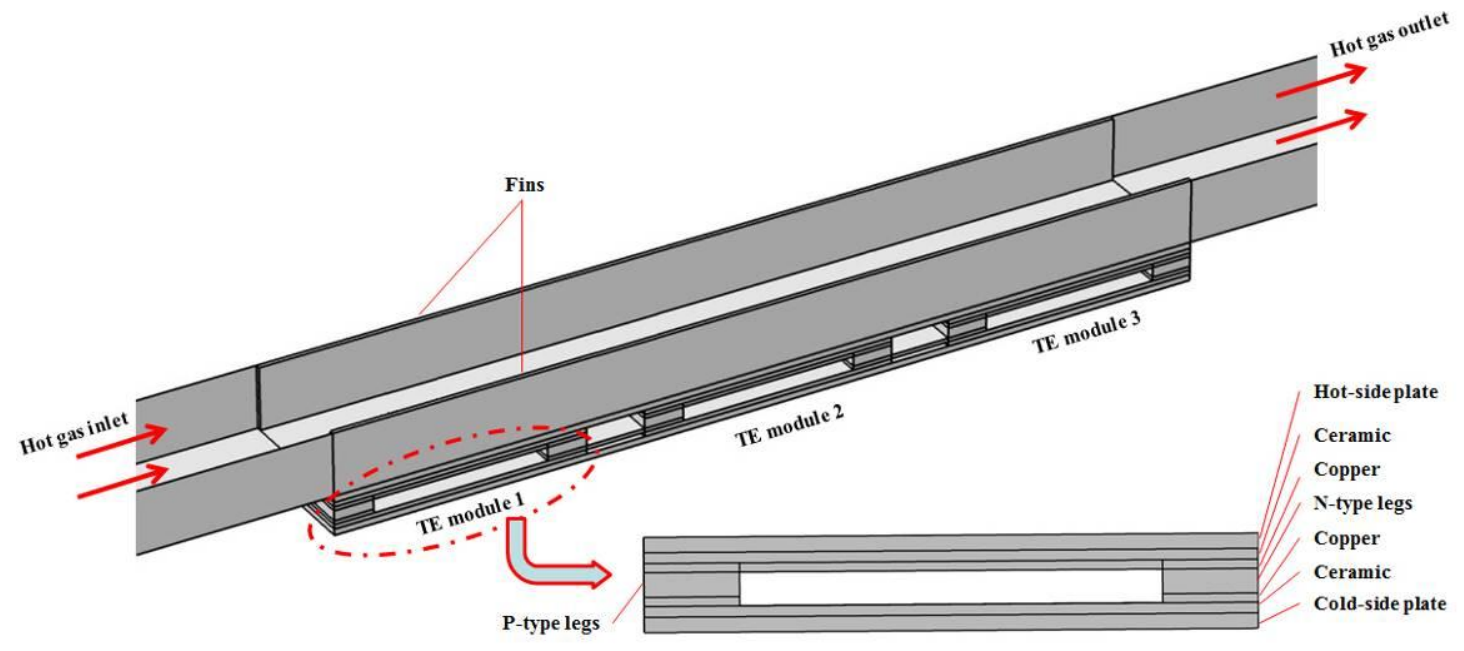

Fig. 1. Physical model of the baseline TEG with a smooth channel. 


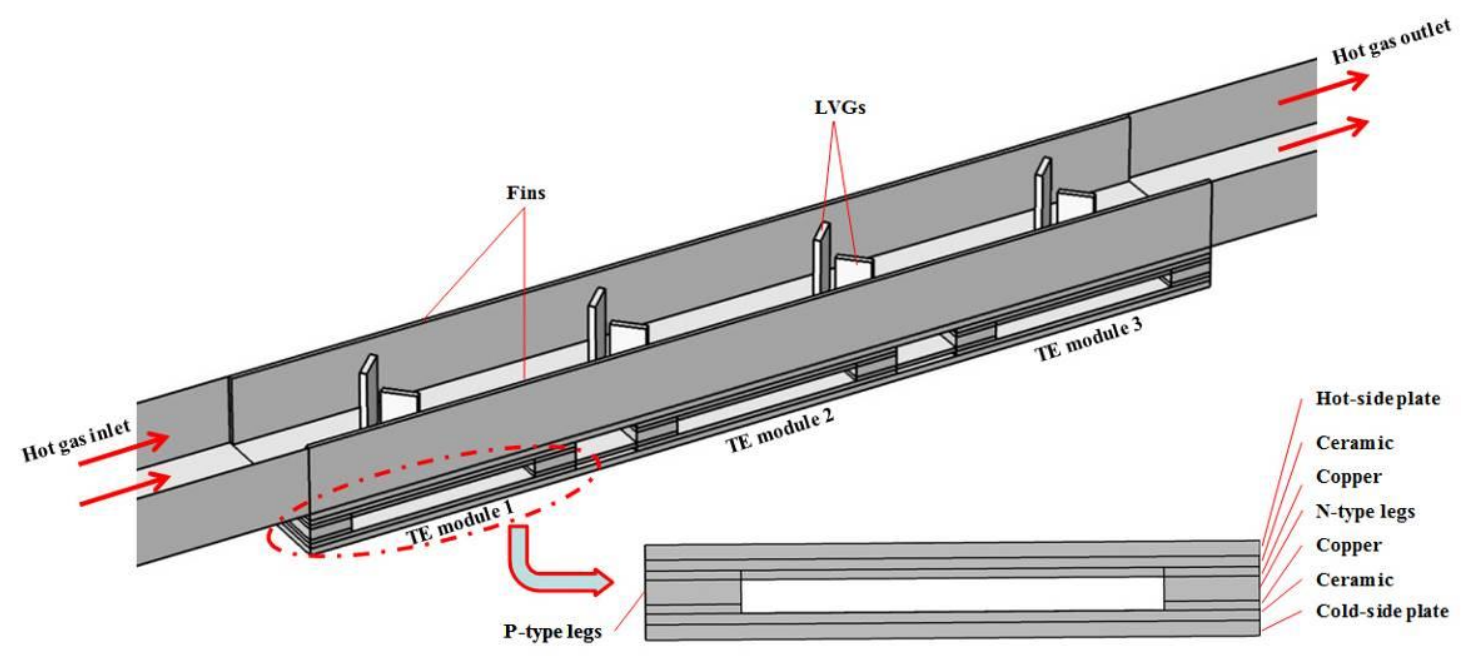

Fig. 2. Physical model of the TEG with LVGs placed in the channel. 


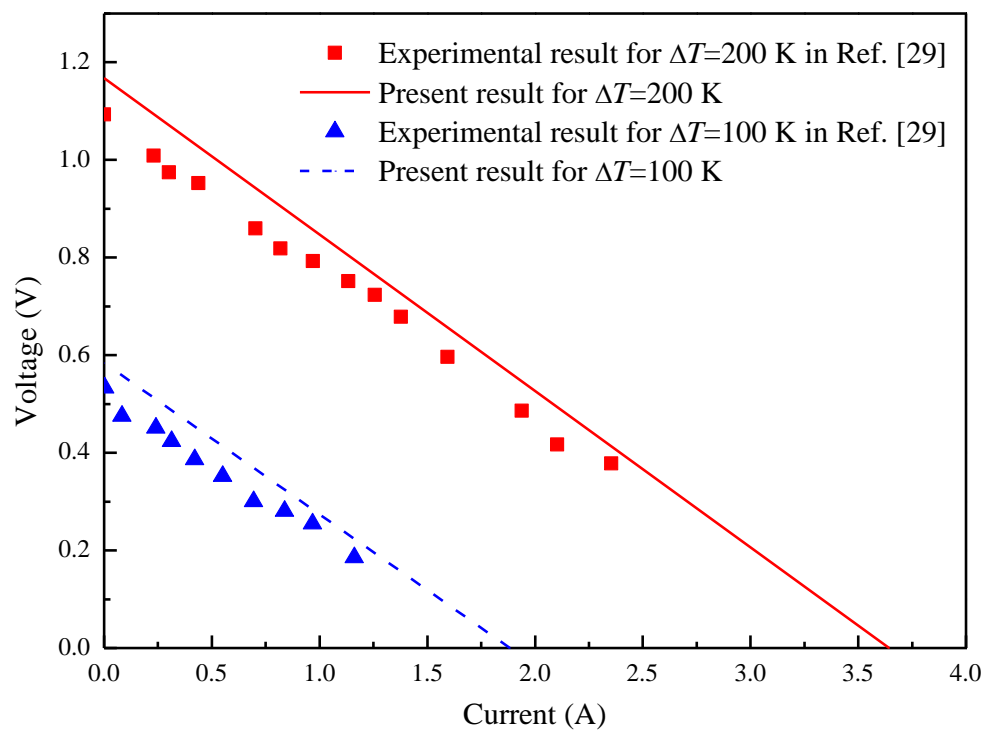

Fig. 3. Validation of the method of using equivalent physical properties for the TE module. 


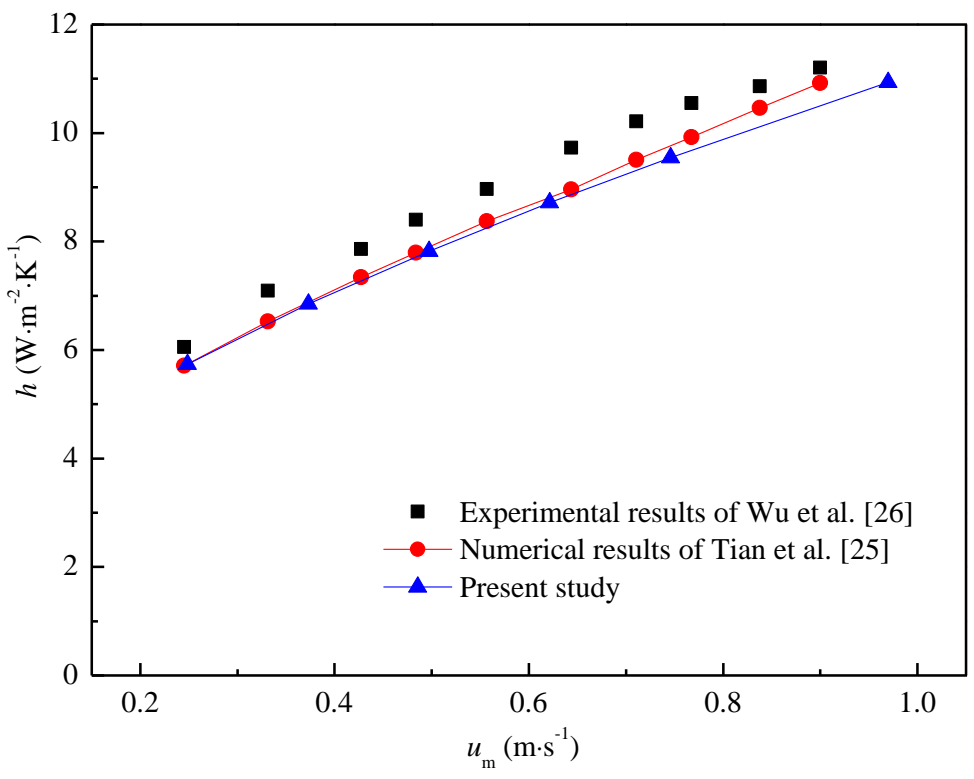

Fig. 4. Validation of the heat transfer performance in a rectangular channel with LVGs. 


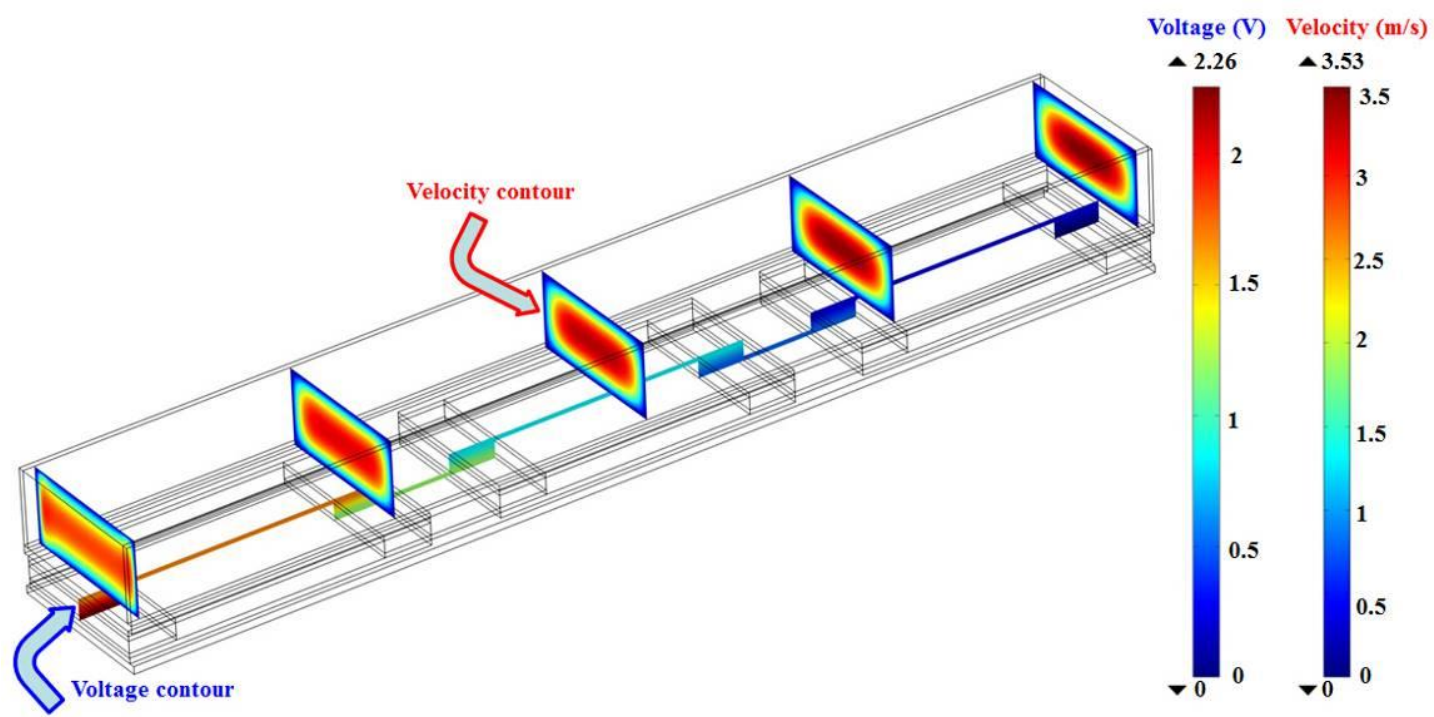

(a) Velocity and voltage distributions of the TEG with smooth channel.

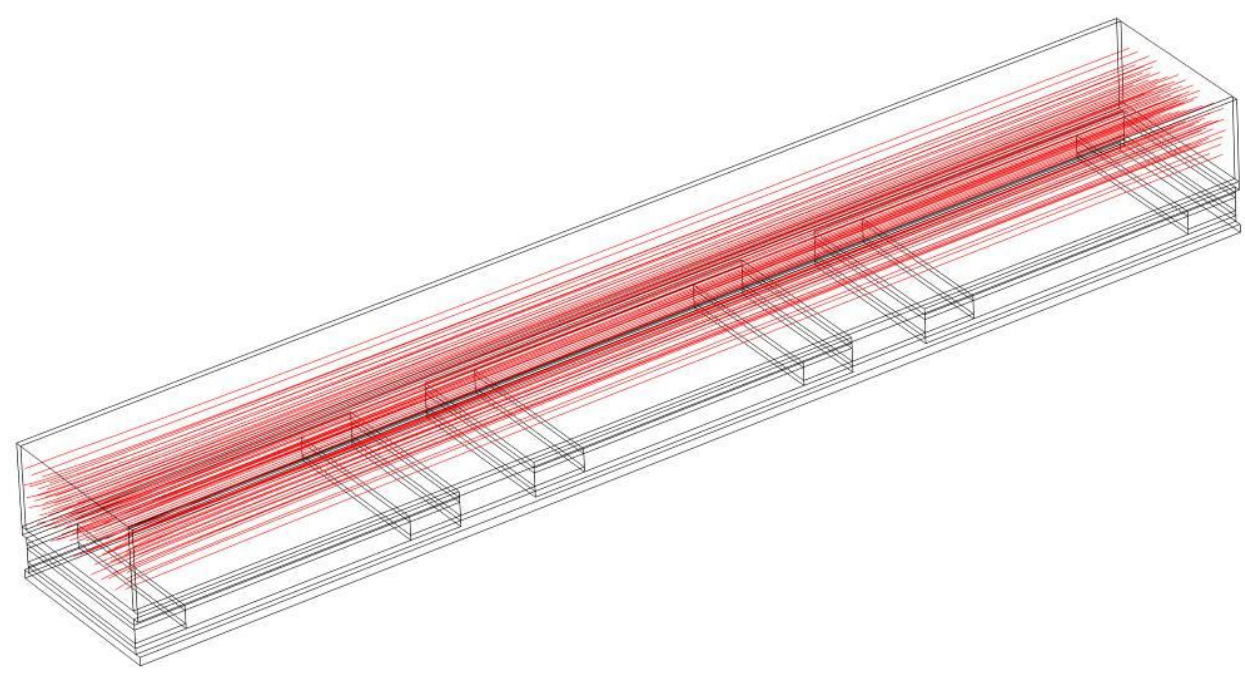

(b) Streamlines of the TEG with smooth channel.

Fig. 5. Velocity, voltage, and streamlines of the TEG with a smooth channel under baseline operating conditions. (a) Velocity and voltage distributions of the TEG with a smooth channel, (b) Streamlines of the TEG with a smooth channel. 


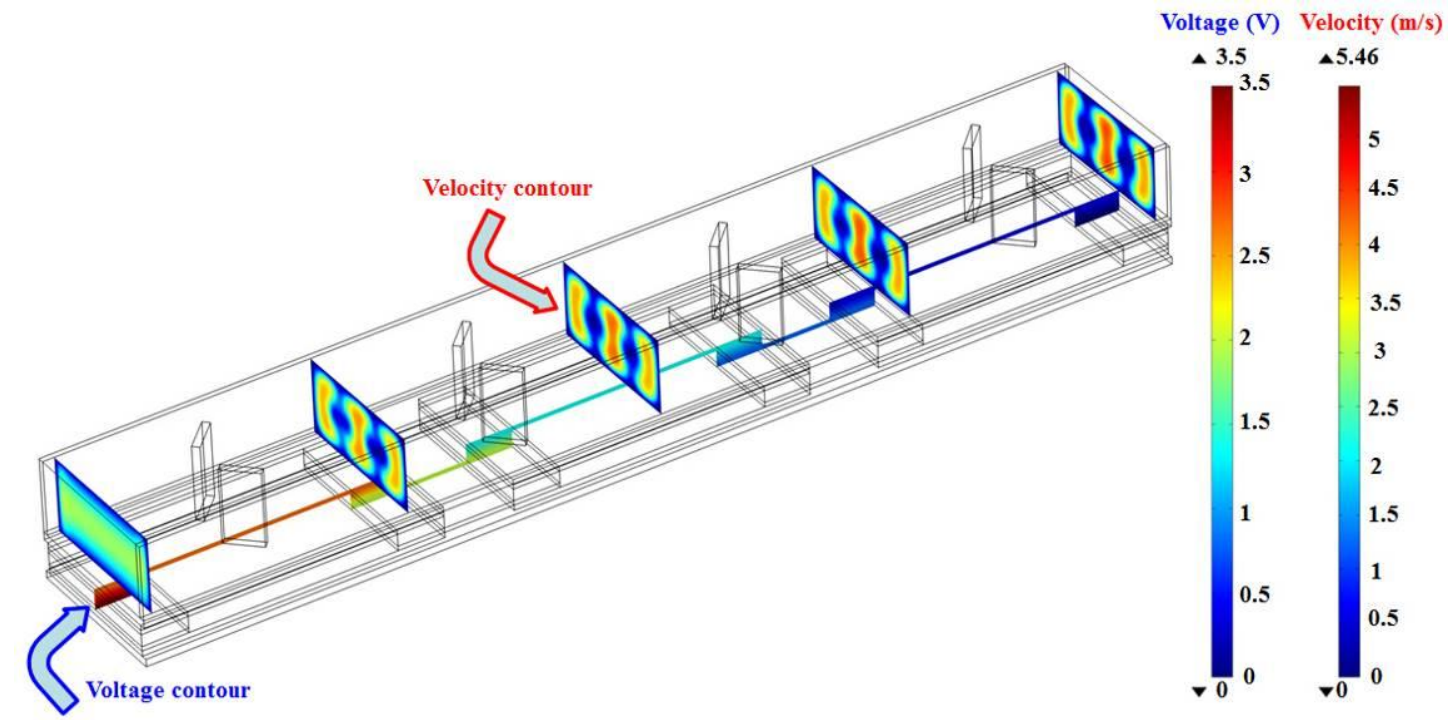

(a) Velocity and voltage distributions of the TEG with LVG channel.

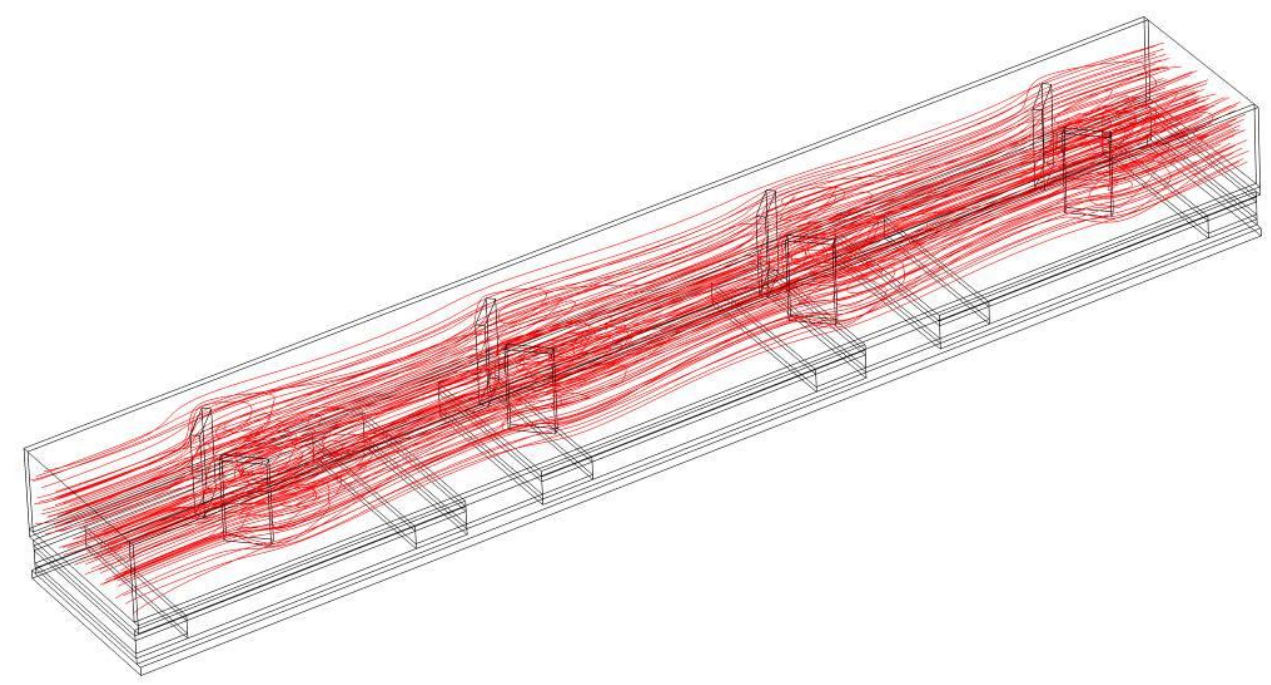

(b) Streamlines of the TEG with LVG channel.

Fig. 6. Velocity, voltage, and streamlines of the TEG with LVGs in the channel under baseline operating conditions. (a) Velocity and voltage distributions of the TEG with LVGs in the channel. (b) Streamlines of the TEG with LVGs in the channel. 


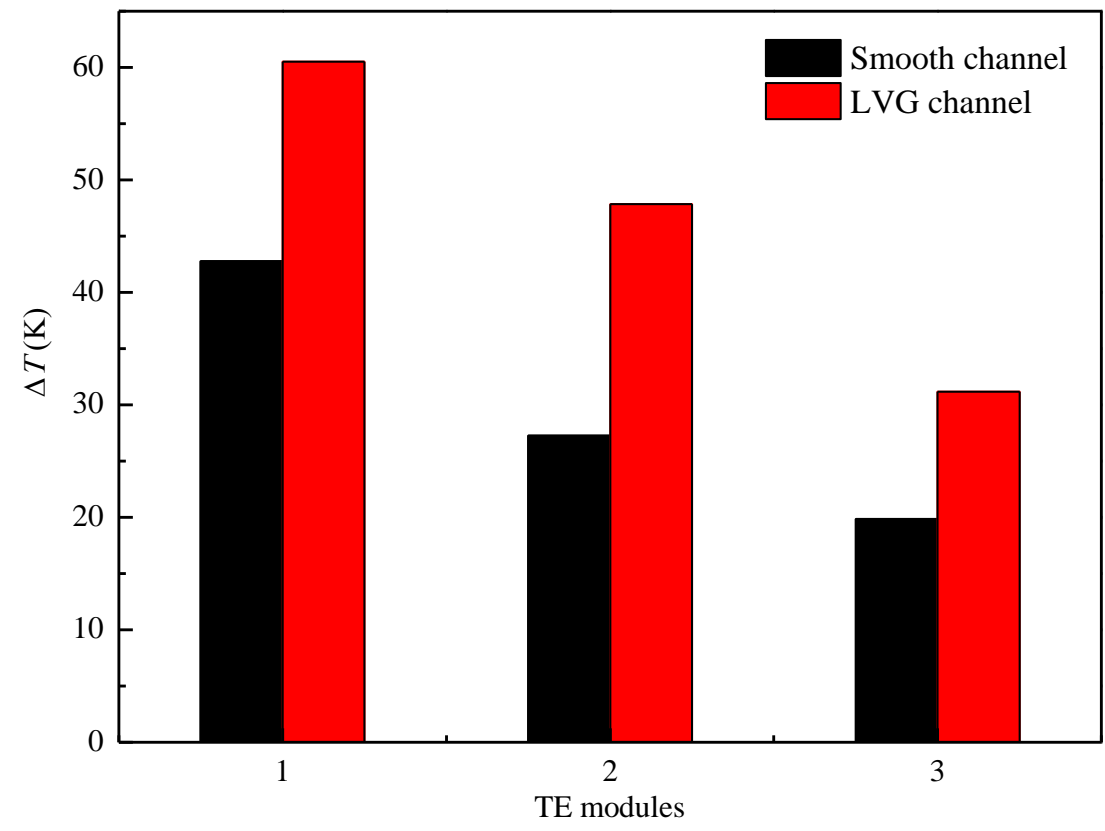

Fig. 7. Comparison of the temperature difference in each TE module under baseline operating conditions. 


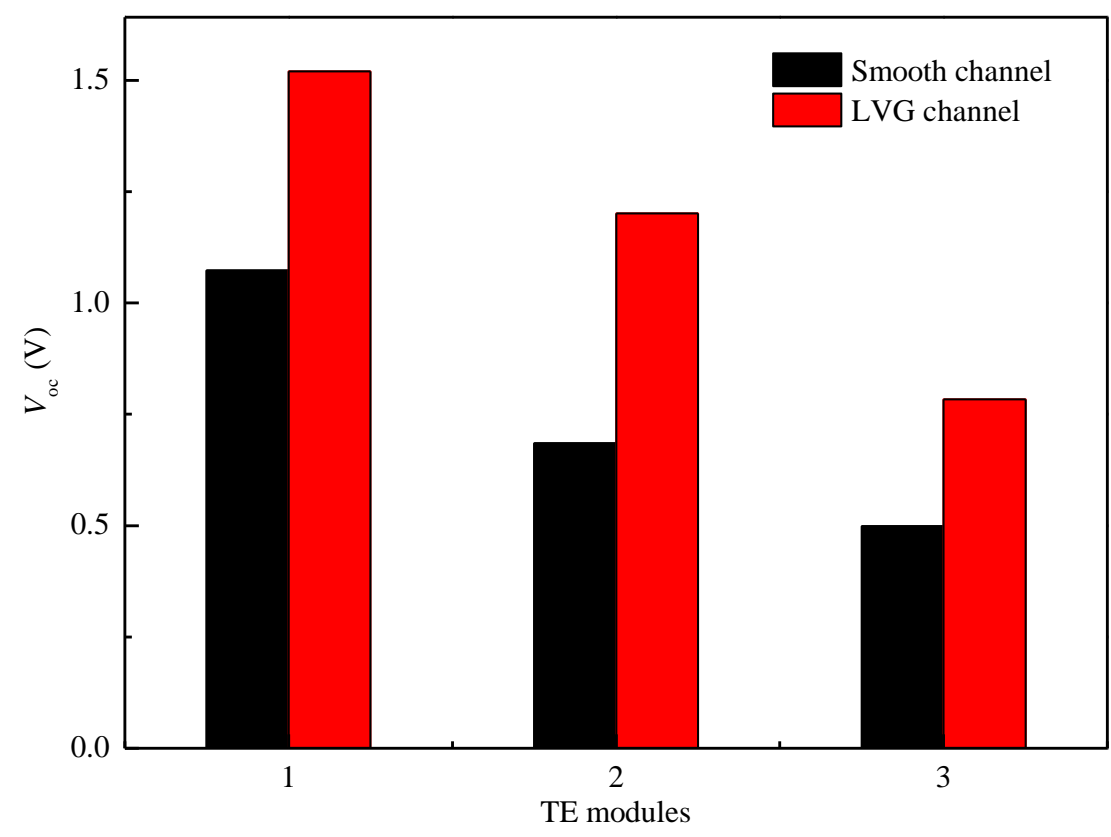

Fig. 8. Comparison of the open circuit voltage in each TE module under baseline operating conditions. 


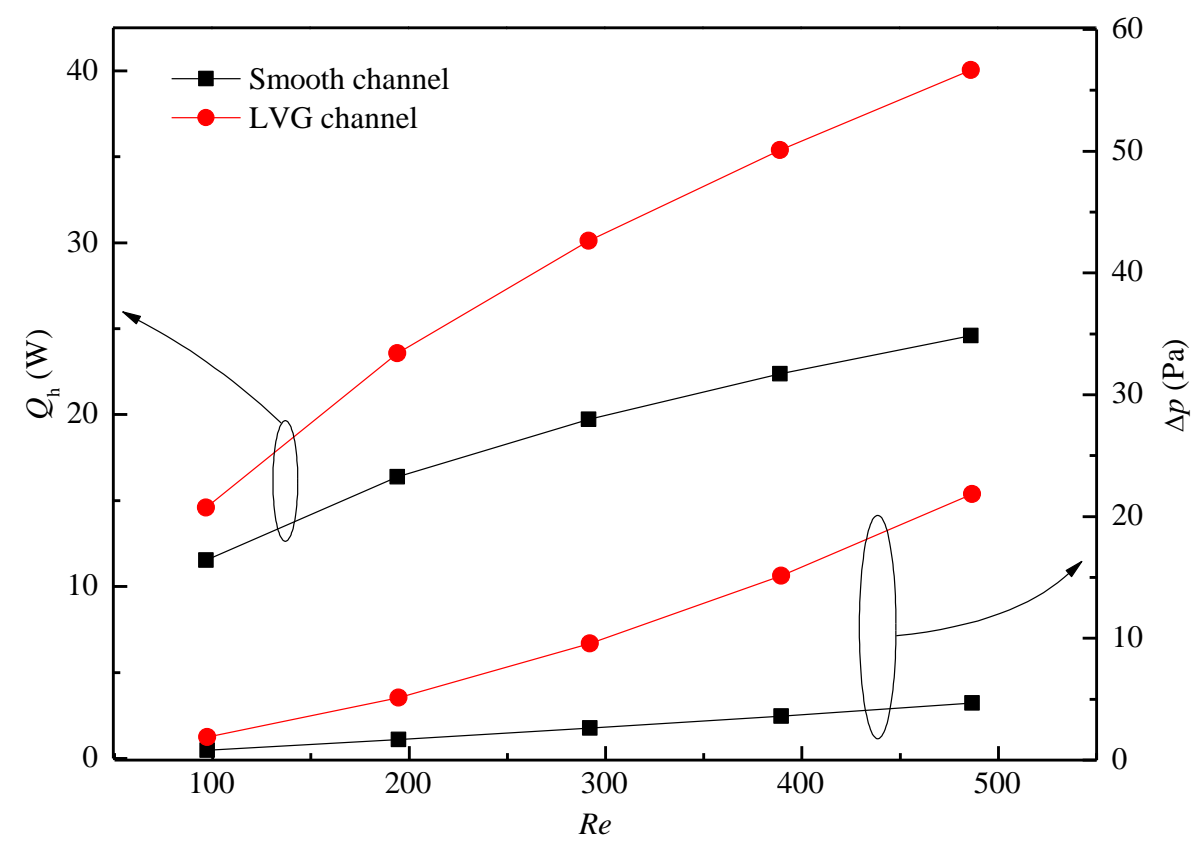

Fig. 9. Effect of Reynolds number on the heat input and pressure drop at $T_{\mathrm{h}, \mathrm{in}}=673.15$ $\mathrm{K}$ and $T_{\mathrm{c}}=363.15 \mathrm{~K}$. 


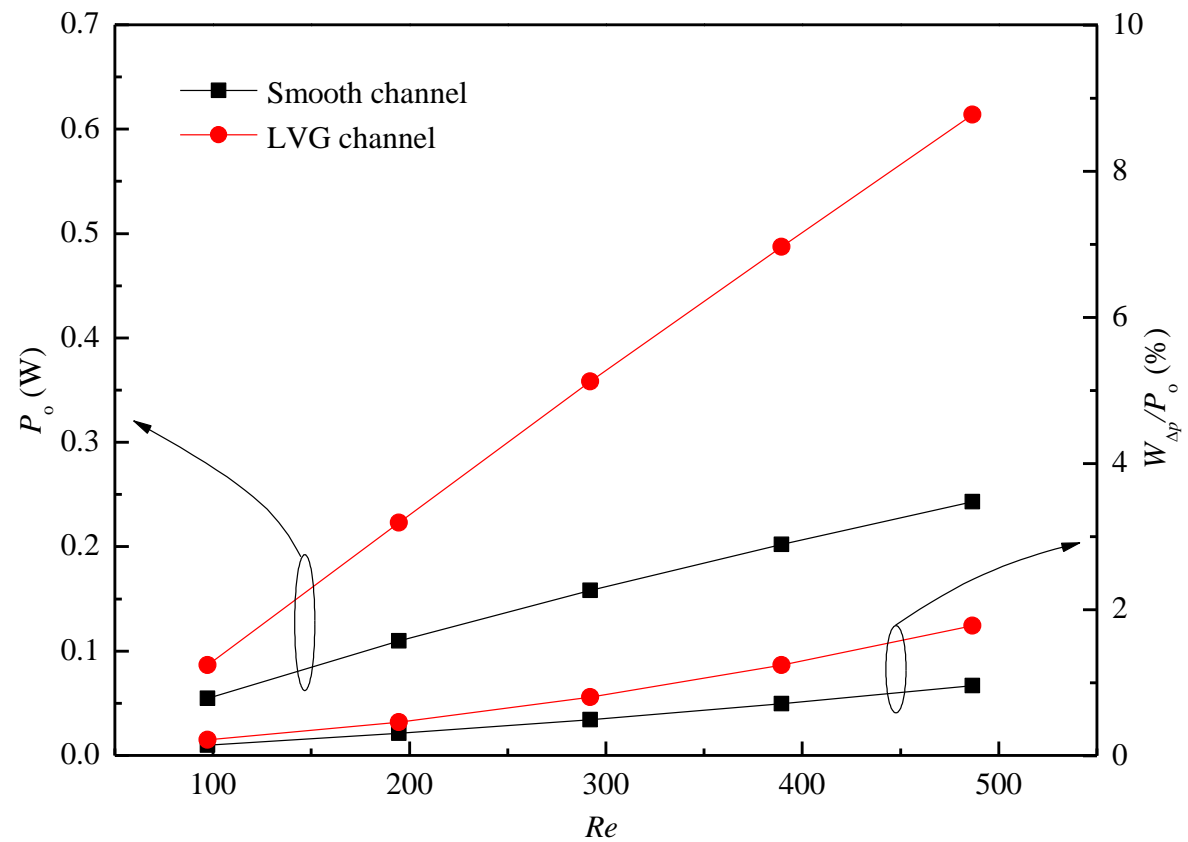

Fig. 10. Effect of the Reynolds number on the total power output and the ratio of pumping power to total power output at $T_{\mathrm{h}, \mathrm{in}}=673.15 \mathrm{~K}$ and $T_{\mathrm{c}}=363.15 \mathrm{~K}$. 


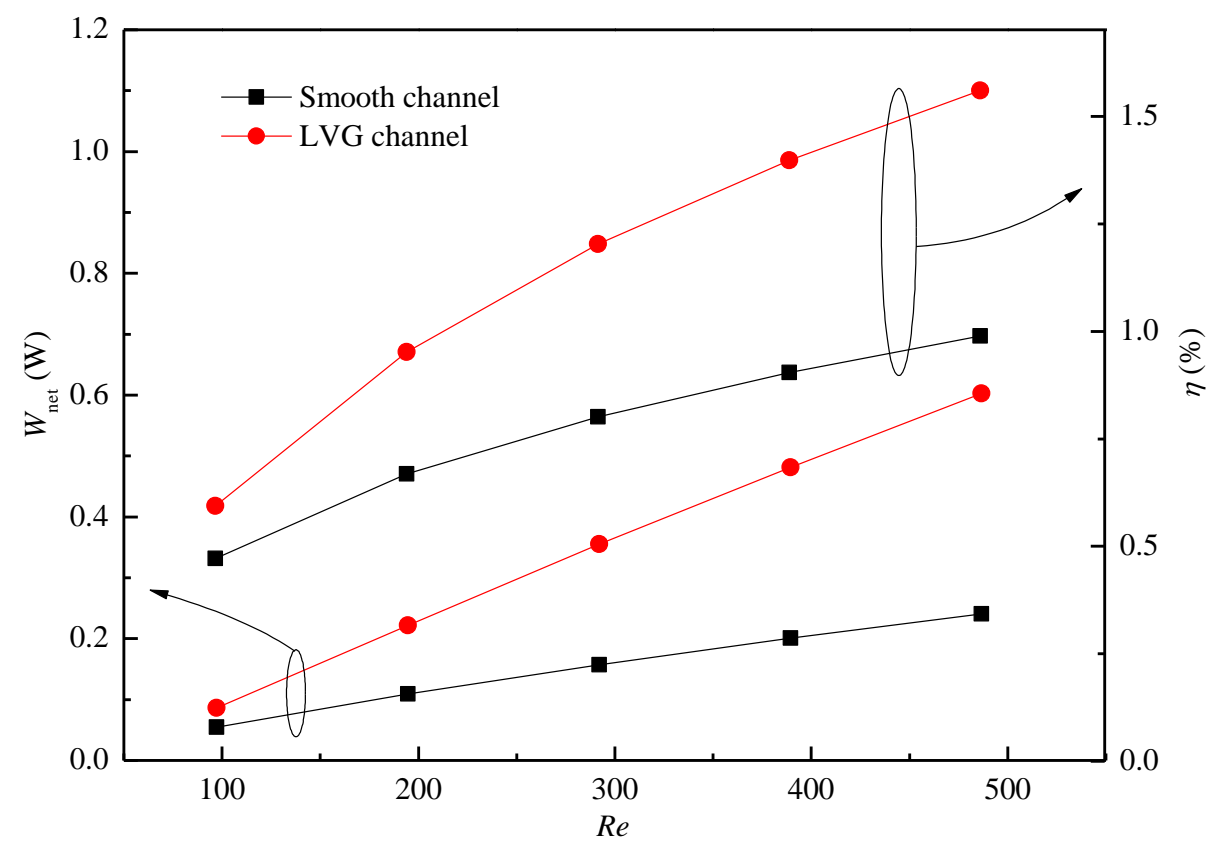

Fig. 11. Effect of the Reynolds number on the net power and thermal conversion efficiency at $T_{\mathrm{h}, \mathrm{in}}=673.15 \mathrm{~K}$ and $T_{\mathrm{c}}=363.15 \mathrm{~K}$. 


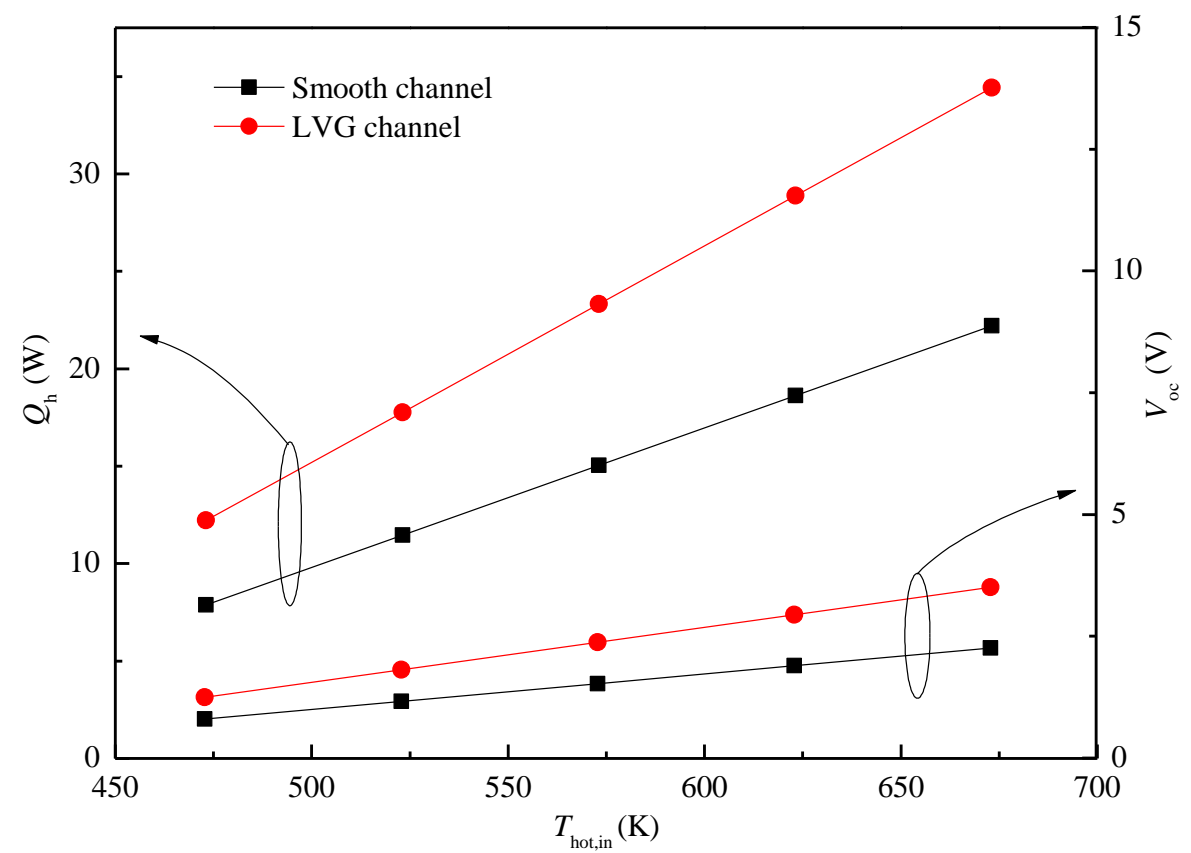

Fig. 12. Effect of the hot-side inlet temperature on the heat input and open circuit voltage at $R e=487$ and $T_{\mathrm{c}}=363.15 \mathrm{~K}$. 


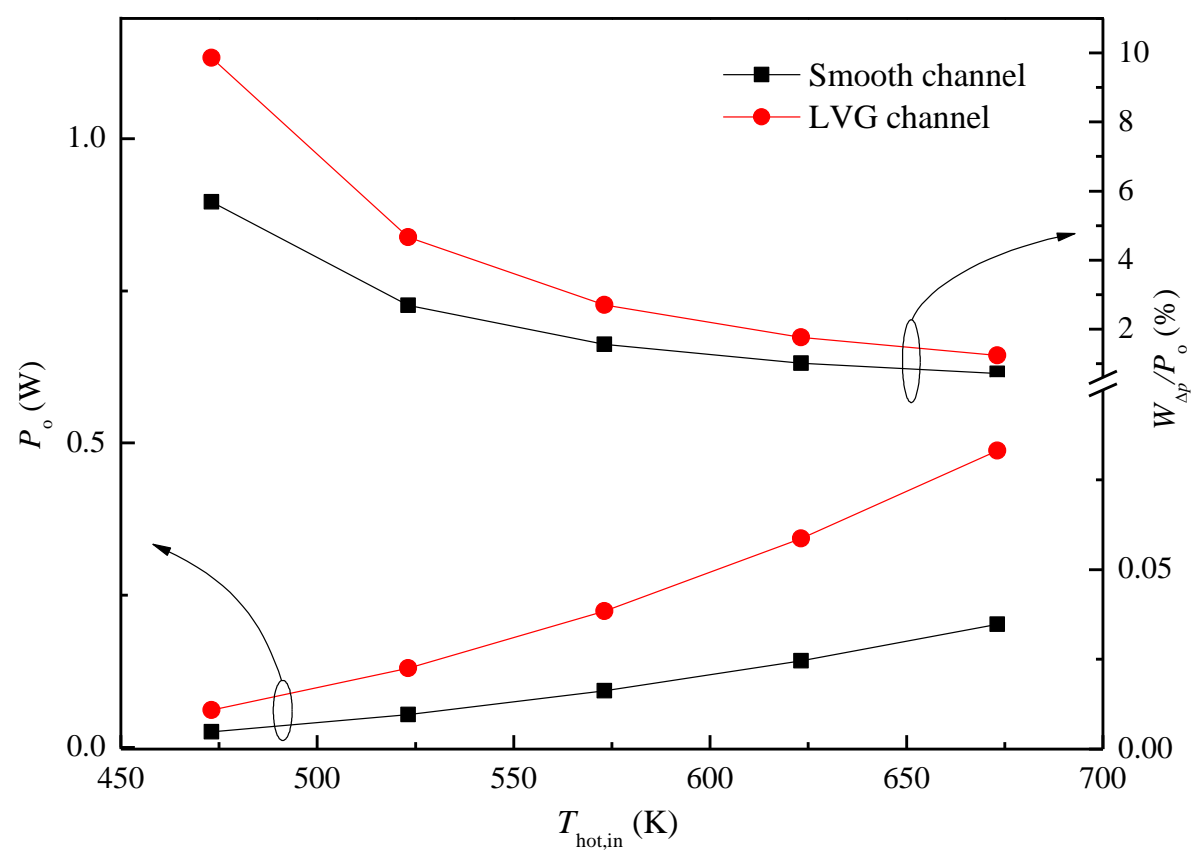

Fig. 13. Effect of the hot-side inlet temperature on the total power output and the ratio of pumping power to total power output at $R e=487$ and $T_{\mathrm{c}}=363.15 \mathrm{~K}$. 


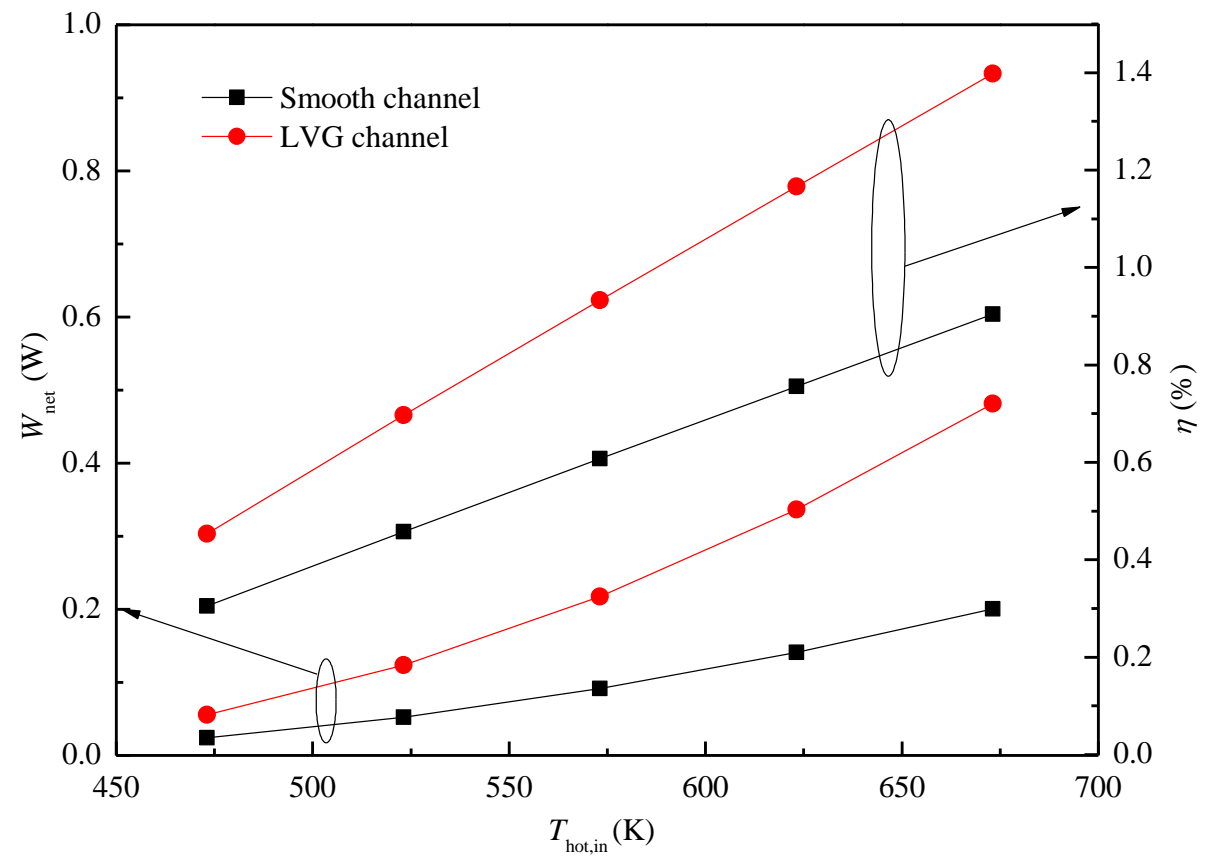

Fig. 14. Effect of the hot-side inlet temperature on the net power and thermal conversion efficiency at $R e=487$ and $T_{\mathrm{c}}=363.15 \mathrm{~K}$. 


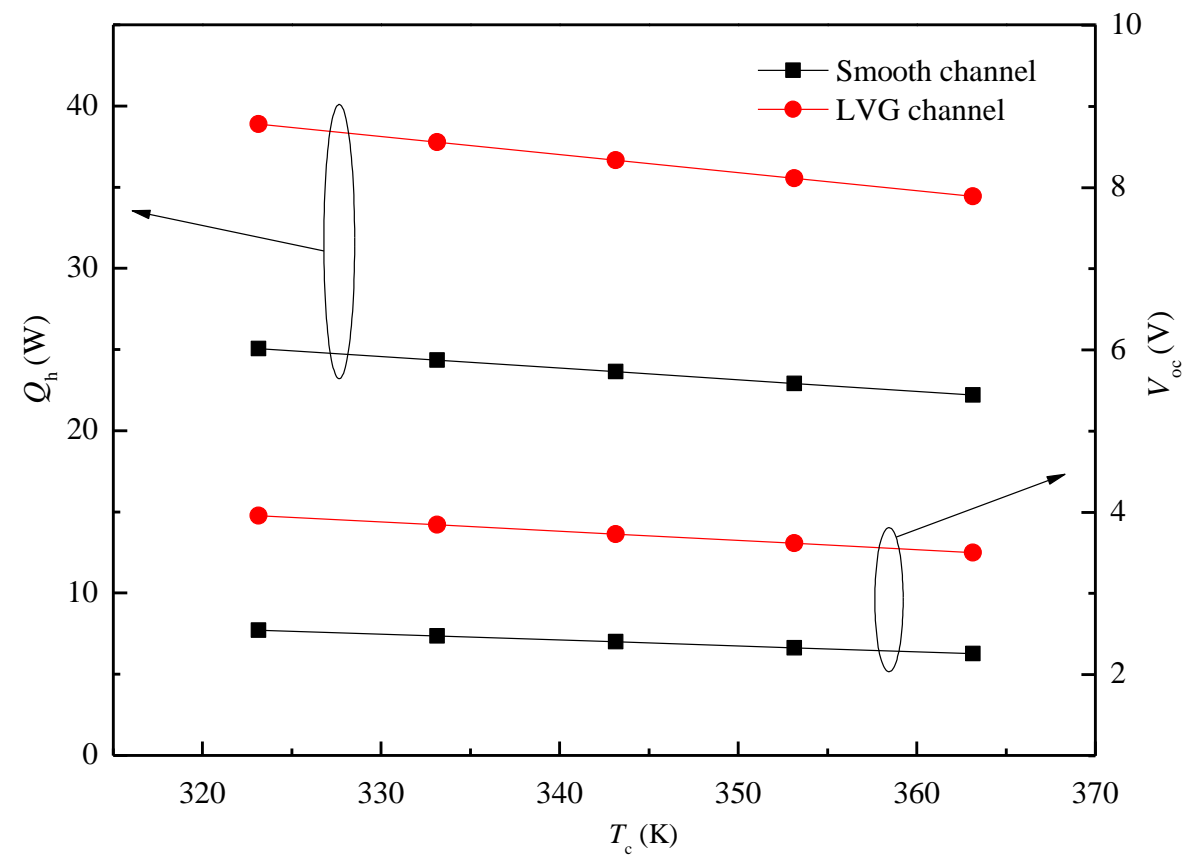

Fig. 15. Effect of the cold-side temperature on the heat input and open circuit voltage at $R e=487$ and $T_{\mathrm{h}, \text { in }}=673.15 \mathrm{~K}$. 


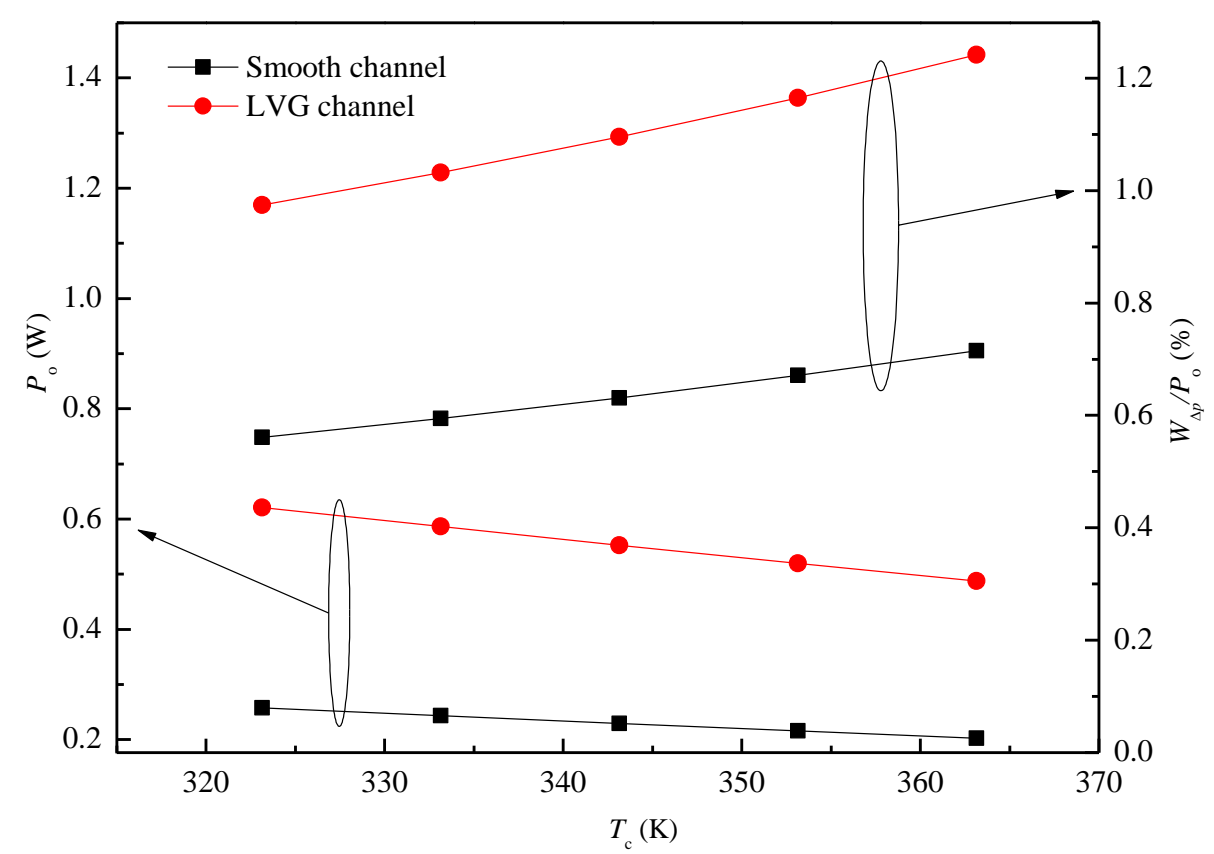

Fig. 16. Effect of the cold-side inlet temperature on the total power output and the ratio of pumping power to total power output at $R e=487$ and $T_{\mathrm{h}, \mathrm{in}}=673.15 \mathrm{~K}$. 


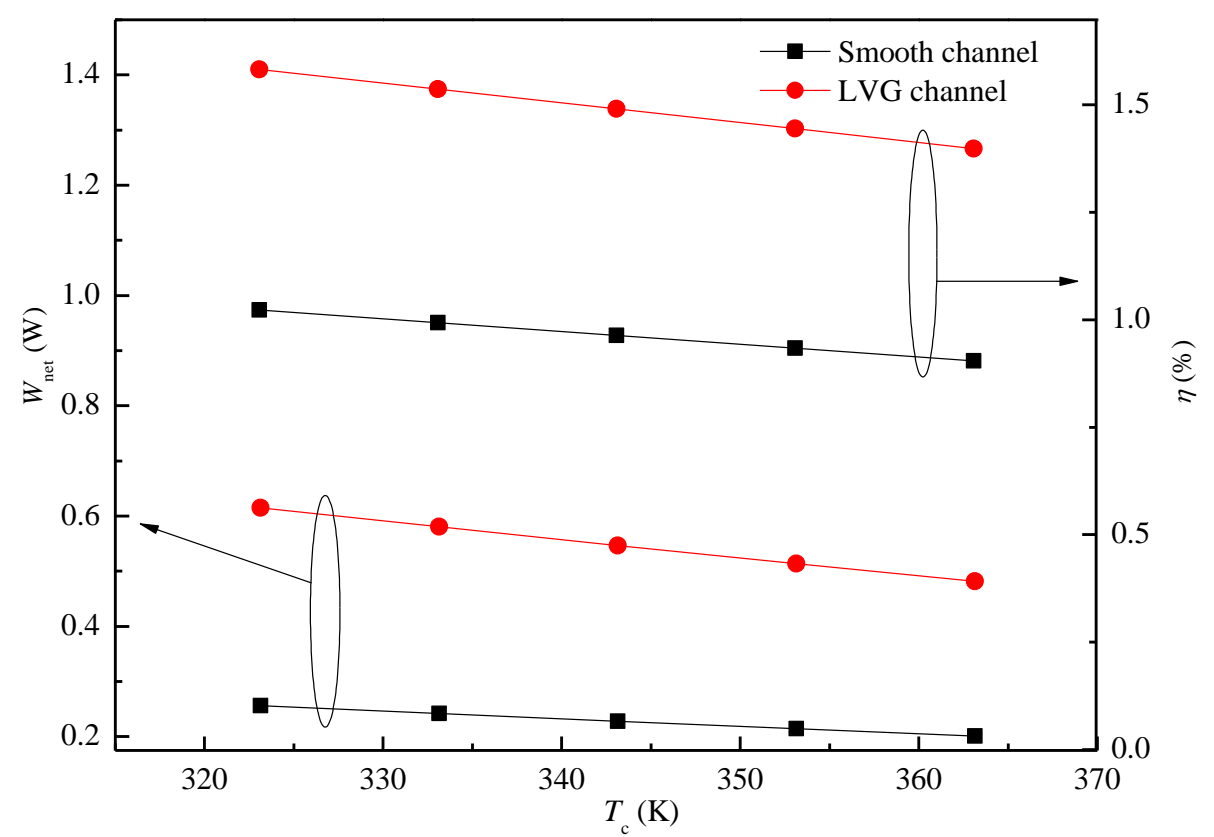

Fig. 17. Effect of the cold-side inlet temperature on the net power and thermal conversion efficiency at $R e=487$ and $T_{\mathrm{h}, \mathrm{in}}=673.15 \mathrm{~K}$. 


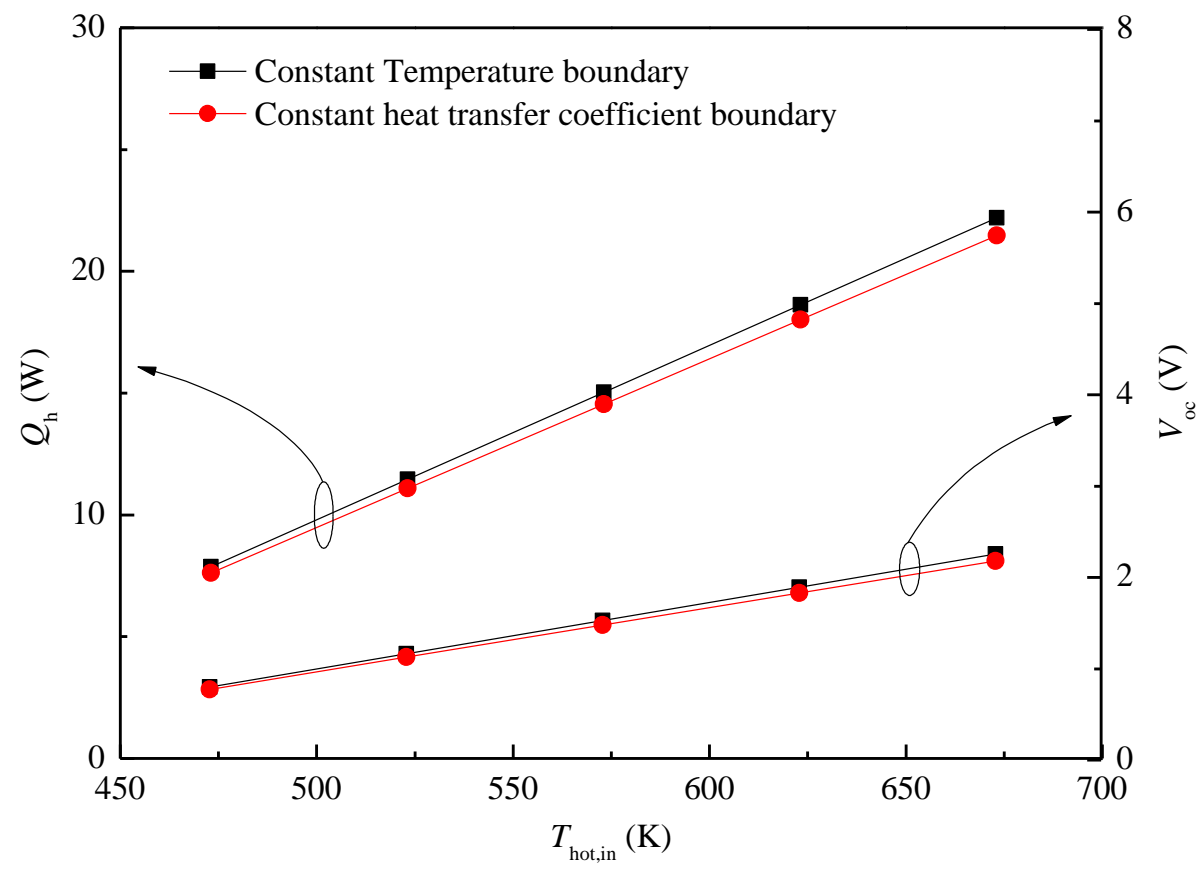

Fig. 18. Effect of the cold-side thermal boundary on the heat input and voltage of the TEG with the smooth channel. 


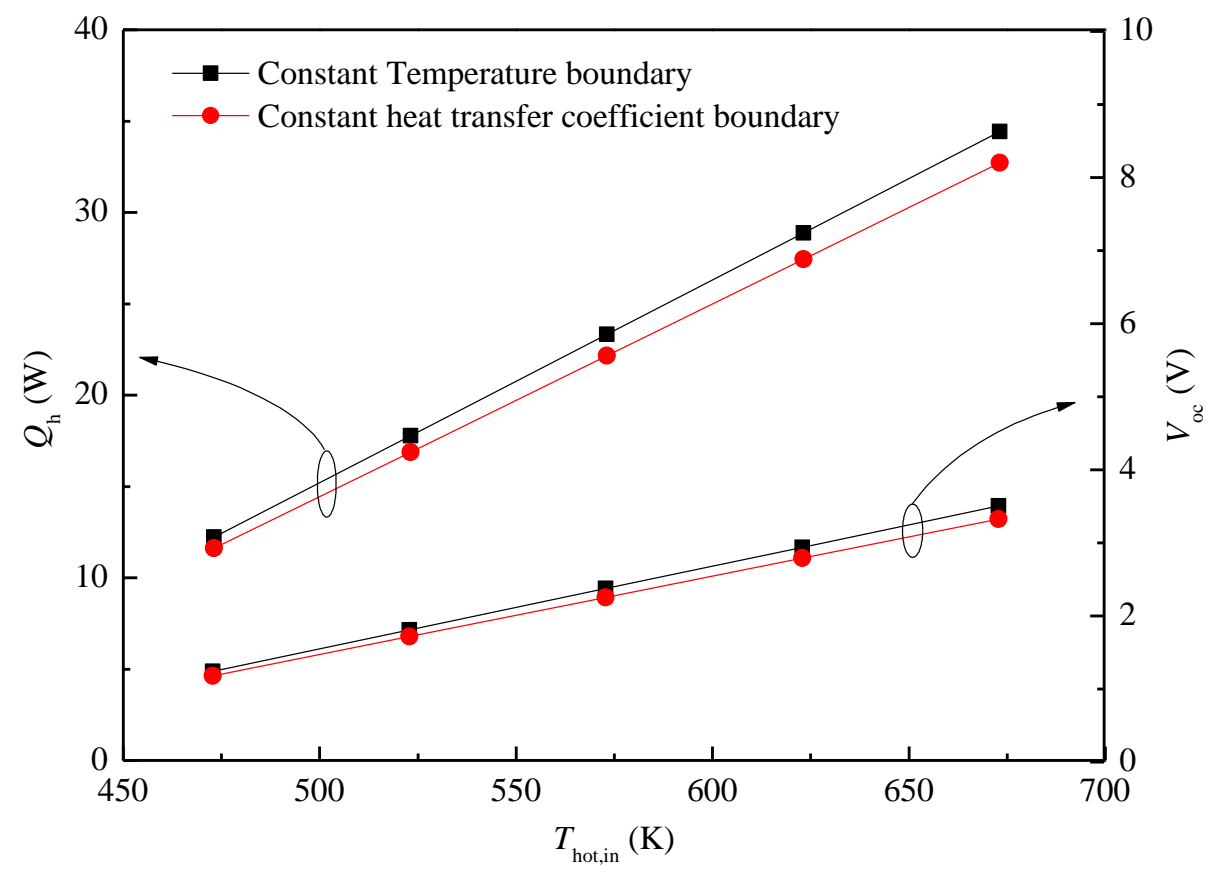

Fig. 19. Effect of the cold-side thermal boundary on the heat input and voltage of the TEG with LVGs in the channel. 